\title{
Magneto-Seebeck coefficient and Nernst coefficient of a hot and dense hadron gas
}

\author{
Arpan Das, ${ }^{1, *}$ Hiranmaya Mishra $\odot,{ }^{2, \dagger}$ and Ranjita K. Mohapatra ${ }^{3, \$}$ \\ ${ }^{1}$ Institute of Nuclear Physics Polish Academy of Sciences, PL-31-342 Kraków, Poland \\ ${ }^{2}$ Theory Division, Physical Research Laboratory, Navrangpura, Ahmedabad 380 009, India \\ ${ }^{3}$ Department of Physics, Banki Autonomous College, Cuttack 754008, India
}

(Received 12 April 2020; revised 18 June 2020; accepted 6 July 2020; published 17 July 2020)

\begin{abstract}
We discuss the thermoelectric effect of hot and dense hadron gas within the framework of the hadron resonance gas model. Using the relativistic Boltzmann equation within the relaxation time approximation we estimate the Seebeck coefficient of the hot and dense hadronic medium with a gradient in temperature and baryon chemical potential. The hadronic medium in this calculation is modeled by the hadron resonance gas (HRG) model with hadrons and their resonances up to a mass cutoff $\Lambda \sim 2.6 \mathrm{GeV}$. We also extend the formalism of the thermoelectric effect for a nonvanishing magnetic field. The presence of magnetic field also leads to a Hall type thermoelectric coefficient (Nernst coefficient) for the hot and dense hadronic matter apart from a magneto-Seebeck coefficient. We find that generically in the presence of a magnetic field the Seebeck coefficient decreases while the Nernst coefficient increases with the magnetic field. At higher temperature and/or baryon chemical potential these coefficients approach to their values at vanishing magnetic field.
\end{abstract}

DOI: $10.1103 /$ PhysRevD.102.014030

\section{INTRODUCTION}

Transport coefficients are important characteristics of thermodynamic systems that determine the evolution of the system toward equilibrium starting from an initial out of equilibrium state. A new state of strongly interacting matter has been reported in the relativistic heavy-ion collision experiments, e.g., at the Relativistic Heavy Ion Collider (RHIC) and the Large Hadron Collider (LHC). This strongly interacting matter so produced is also expected to achieve local thermal equilibrium within a few Fermi timescale after the collision, which is supported by the fact that relativistic hydrodynamical modeling of the strongly interacting medium successfully explains the particle spectra coming out of the medium after hadronization. Similar to other thermodynamic systems transport coefficients of the strongly interacting plasma are of utmost importance for a comprehensive understanding of quantum chromodynamics (QCD) in the nonperturbative regime. In the dissipative relativistic hydrodynamical model as well as in

\footnotetext{
*arpan.das@ifj.edu.pl

†hm@prl.res.in

ॠranjita.iop@gmail.com
}

Published by the American Physical Society under the terms of the Creative Commons Attribution 4.0 International license. Further distribution of this work must maintain attribution to the author(s) and the published article's title, journal citation, and DOI. Funded by SCOAP ${ }^{3}$. the transport simulations of the hot and dense medium these transport coefficients, e.g., shear and bulk viscosity, etc. play important roles. It has been argued that the strongly interacting medium produced at RHIC is the most perfect fluid in nature having the smallest value of shear viscosity to entropy ratio $(\eta / s)$ known to us [1-3]. Apart from the shear viscosity another important transport coefficient which play an important role in the hydrodynamical evolution of non conformal field theories is the bulk viscosity $\zeta$ [4-12]. Although at asymptotically high temperature relative to $\Lambda_{\mathrm{QCD}}$ strongly interacting plasma can be approximated with the conformal equation of state $(\mathcal{E}=3 P)$, lattice $\mathrm{QCD}$ simulations shows a nonmonotonic behavior of both $\eta / s$ and $\zeta / s$ near the critical temperature $T_{c}$. [6-12]. It is important to note that the bulk viscosity encodes the conformal measure, $(\mathcal{E}-3 P) / T^{4}$ of the system and nonvanishing bulk viscous coefficient indicates deviation from conformality of the strongly interacting matter. A new dimension has been added to the study of the strongly interacting plasma is the prediction of the generation of a strong magnetic field in the noncentral heavyion collisions. Conservative estimates indicate that the magnitude of the magnetic field can be as large as of several $m_{\pi}^{2}$, at least in the initial stage at RHIC energies [13-16]. The strength of the magnetic field in the initial stage can be large with respect to $\Lambda_{\mathrm{QCD}}$, but in the absence of any thermalized conducting medium, this large magnetic field will quickly vanish. If the thermalization time of the strongly interacting medium is small then it can be argued 
that due to finite electrical conductivity some fraction of the initial large magnetic field may sustain in the conducting plasma [17-35]. Certainly, it is not known what is the exact amount of magnetic field that can be sustained in the medium, but taking an optimistic approach many investigations are going on to understand novel effects of magnetic field on QCD plasma. One of such effect is the $C P$-violating effects, e.g., chiral magnetic effect and chiral vortical effect [36]. The success of hydrodynamical modeling of the QCD plasma inspires a deeper understanding of hydrodynamical behavior in the presence of a magnetic field. In this context, magnetohydrodynamic simulations have been used to study the flow coefficient of the strongly interacting matter produced in heavy-ion collisions [17,18]. This apart various approaches like perturbative QCD (pQCD), and different QCD inspired effective models, etc. have been used to estimate various transport coefficients for the QCD matter both in the absence and in the presence of magnetic field [37-71]. Another transport coefficient which becomes important in the presence of temperature gradient at finite baryon density is the thermal conductivity [72,73]. The effect of the magnetic field on thermal conductivity of hot and dense hadron gas has been discussed in the literature [68].

In the present article, we investigate thermoelectric behavior in the presence of the magnetic field, specifically the magneto-Seebeck coefficient and Nernst coefficient of the hadronic medium produced in the subsequent evolution of the partonic medium formed in heavy-ion collisions. Due to nonvanishing thermoelectric coefficients, a temperature gradient in a conducting medium gets converted into an electrical current and vice versa. The Seebeck coefficient is a measure of the electric field produced in a conducting medium due to a temperature gradient and it is formally defined by the "open circuit" condition, i.e., when the electric current is set to zero [74,75]. In condensed matter systems Seebeck effect has been investigated extensively, e.g., in superconductors [76,77], graphene-superconductor junction [78], Majorana bound state coupled to a quantum dots [79], high temperature cuprates [80], superconductorferromagnetic tunnel junctions [81], low dimensional organic metals [82] etc. Following the formalism developed in condensed matter systems as given in Refs. [83,84], earlier some of us had studied the Seebeck coefficient in the absence of a magnetic field for the hot and dense hadronic matter within the framework of the hadron resonance gas model [66]. The present investigation differs from our earlier work in the following ways: in Ref. [66] we had investigated thermoelectric effect for a system that has a temperature gradient but we had considered spatially uniform chemical potential. Here we consider a temperature gradient as well as a gradient in the baryon chemical potential to estimate the thermoelectric coefficients. Due to Gibbs-Duhem relation, one can relate the temperature gradient with the gradient in the baryon chemical potential in the QCD medium [4]. It is important to note that, contrary to a nonrelativistic system where heat current is defined with respect to Fermi surface, in a relativistic system thermal current can only be defined in the presence of conserved number current, e.g., for strongly interacting matter one generally defines heat current with respect to the baryon current due to the fact that baryon number is conserved in QCD. This is an important difference with respect to the definition of heat current in the nonrelativistic systems. These differences between the two formalisms are very important and due to these differences, the nature of estimated thermoelectric coefficients will be very different which we will show later in the formalism as well as in the result section. Further, we have extended the formalism of the thermoelectric effect for the nonvanishing magnetic field and estimate the magneto-Seebeck coefficient and the Nernst coefficient. Unlike the Seebeck coefficient, the Nernst coefficient is a Hall-type thermoelectric coefficient having nonvanishing value only for a finite magnetic field. If a conducting medium is subjected to a magnetic field in the presence of temperature gradient perpendicular to the magnetic field then the Nernst coefficient is the manifestation of electric current normal to both magnetic field and temperature gradient.

In the condensed matter systems in the absence of a magnetic field nonvanishing Seebeck coefficient is ensured by the presence of temperature gradient only as because in these systems only one type of charge carriers are available e.g., either electrons or holes. Also for the electron-ion plasma where the different mobility of electrons and ions can give rise to a nonvanishing value of the Seebeck coefficient. On the other hand for an electron-positron plasma just having a temperature gradient does not guaranty any thermoelectric current in the absence of a magnetic field due to the cancellation of electric current due to particles and antiparticles. For strongly interacting plasma since heat current can only be defined with reference to the net baryon current, the Seebeck coefficient is only meaningful at finite baryon chemical potential. It is important to note that although the Seebeck coefficient is associated with electric current due to temperature gradient, due to Onsager relations thermoelectric coefficients associated with electrical current and the thermoelectric coefficients associated with the heat current are related, e.g., Peltier coefficient and Seebeck coefficient are not independent [85]. At finite baryon chemical potential, the number of baryons and antibaryons are different and a net thermoelectric current driven by the temperature gradient can be produced. For heavy-ion collision experiments at RHIC and LHC the medium produced is expected to have very small net baryon number density, however, the heavy-ion collisions at Facility for Antiproton and Ion Research (FAIR) at Darmstadt [86] and in Nuclotron-based Ion Collider fAcility (NICA) at Dubna [87] one expects a baryon-rich medium. In these low energy experiments, the 
thermalization of the strongly interacting medium is expected with finite baryon density. All the conditions for a nonvanishing thermoelectric effect, e.g., temperature gradient, nonvanishing baryon number, etc., allows us to study thermoelectric effects in these low energy heavy-ion collision experiments. Description of thermoelectric coefficients becomes more complicated in the presence of a magnetic field and in this investigation we have discussed in detail the effect of the magnetic field on the Seebeck coefficient and the Nernst coefficient. We have found that generically in the presence of magnetic field Seebeck coefficient of hot and dense hadron gas decreases. On the other hand, the Nernst coefficient increases with the magnetic field. Here we have assumed that in the hadronic system the strength of the magnetic field is small and it is not a dominant scale. In the present approach, therefore, we attempted to estimate the transport coefficients where the phase space and the single-particle energies are not affected by the magnetic field through Landau quantization as in Refs. [67-69,71]. On the other hand, the effect of the magnetic field enters through the cyclotron frequency of the individual hadrons.

In the context of heavy ion collision, the hadronic phase of the QCD plasma can be successfully described by the hadron resonance gas (HRG) model, at chemical freeze-out [88-90]. In the simplest approximation the strange and nonstrange particles freeze out in the same manner and in this simple case HRG model can be described by only two parameters i.e., temperature $(T)$ and baryon chemical potential $\left(\mu_{B}\right)$. It has been argued that thermodynamics of strongly interacting hadronic systems can be approximated by a system of noninteracting hadrons and its resonances, where the interactions between different hadrons are encoded as the resonances [91,92]. HRG model has been explored regarding thermodynamics of hadronic medium [93,94], conserved charge fluctuations [95-100] as well as transport coefficients for hadronic matter[20,38-41,43-49,51-58,61,62]. Subsequently, the ideal HRG model has been improved upon, e.g., considering excluded volume due to the finite size of the hadrons $[62,101]$. We would like to mention here that, although some of us studied the Seebeck coefficient for the hadronic medium using the formalism originally developed for condensed matter system, here we study the magnetoSeebeck coefficient and Nernst coefficient for the hadronic system using the formalism of thermoelectric effect compatible with relativistic systems.

This paper is organized as follows, in Sec. II we introduce the formalism to estimate Seebeck coefficient in the absence of a magnetic field for a relativistic system which will be followed by the formalism of magnetoSeebeck coefficient and Nernst effect in Sec. III. In Sec. IV we present and discuss the results for the magneto-Seebeck coefficient and the Nernst coefficient. Finally, we summarize our work with an outlook in the conclusion section.

\section{BOLTZMANN EQUATION IN RELAXATION TIME APPROXIMATION AND THERMOELECTRIC EFFECT}

We first discuss here the thermoelectric effect in the absence of a magnetic field. We consider here the linearized Boltzmann equation in relaxation time approximation. The thermal equilibrium is achieved locally due to strong interaction and the external electromagnetic field acts as perturbation which takes the system slightly away from equilibrium. Under this approximation in the Boltzmann equation the distribution function can be expanded around the equilibrium distribution function and one can keep terms up to linear order in the deviation from the equilibrium distribution function. Spatial dependence of the distribution functions appears due to spatial dependence of temperature $(T)$ and baryon chemical potential $\left(\mu_{B}\right)$, in local thermodynamic equilibrium. The relativistic Boltzmann transport equation (RBTE) in the presence of an electromagnetic field for a single hadron species can be expressed as $[71,83,84,102,103]$,

$$
p_{a}^{\mu} \frac{\partial f_{a}}{\partial x^{\mu}}+q_{a} F^{\mu \nu} p_{a, \nu} \frac{\partial f_{a}}{\partial p_{a}^{\mu}}=\mathcal{C}\left[f_{a}\right],
$$

here $F^{\mu \nu}$ is the electromagnetic field strength tensor which in general contains the electric field $(\vec{E})$ and the magnetic field $(\vec{B}), q_{a}$ is the electric charge of the particle species " $a$ " and $\mathcal{C}\left[f_{a}\right]$ is the collision integral. Here $f_{a}$ denotes the off equilibrium distribution function. The equilibrium distribution function will be denoted by $f_{a}^{(0)}$ and is given by $\left.f_{a}^{(0)}(\vec{r}, \vec{p})=\left[\exp \left\{\left(p_{a}^{\nu} u_{\nu}-b_{a} \mu_{B}(\vec{r})\right) / T(\vec{r})\right\} \pm 1\right)\right]^{-1}$. Here \pm are for fermions and bosons respectively and $b_{a}$ denotes the baryon number. In the relaxation time approximation (RTA) the collision integral can be written as [67,69,71],

$$
\mathcal{C}\left[f_{a}\right] \simeq-\frac{p_{a}^{\mu} u_{\mu}}{\tau_{a}}\left(f_{a}-f_{a}^{(0)}\right) \equiv-\frac{p_{a}^{\mu} u_{\mu}}{\tau_{a}} \delta f_{a} .
$$

In Eq. (2), $u^{\mu}$ is the fluid four velocity. In the local rest frame the four velocity takes the form $(1, \overrightarrow{0})$. Here $\delta f_{a}$ denotes the deviation from equilibrium and $\tau_{a}$ denotes the relaxation time of the particle species " $a$ ". Using Eq. (2), in the local rest frame, the relativistic Boltzmann equation as given in Eq. (1) in the absence of a magnetic field can be expressed as $[4,71,102,103]$,

$$
\frac{\partial f_{a}}{\partial t}+\vec{v}_{a} \cdot \vec{\nabla} f_{a}+q_{a} \vec{E} \cdot \frac{\partial f_{a}}{\partial \vec{p}_{a}}=-\frac{\delta f_{a}}{\tau_{a}} .
$$

In a static case, one can assume that both $f_{a}$ and $f_{a}^{(0)}$ do not depend on time explicitly [71]. Therefore, for the static case and in the linear order in the deviation from the equilibrium distribution, the relativistic Boltzmann equation get reduced to, 


$$
\begin{aligned}
\vec{v}_{a} \cdot \vec{\nabla} f_{a}^{(0)}+q_{a} \vec{E} \cdot \frac{\partial f_{a}^{(0)}}{\partial \vec{p}_{a}} & =-\frac{f_{a}(\vec{r}, \vec{p})-f_{a}^{(0)}(\vec{r}, \vec{p})}{\tau_{a}(\vec{p})} \\
& \equiv-\frac{\delta f_{a}(\vec{r}, \vec{p})}{\tau_{a}(\vec{p})}
\end{aligned}
$$

The equilibrium distribution function satisfies,

$$
\begin{gathered}
\frac{\partial f_{a}^{(0)}}{\partial \vec{p}_{a}}=\vec{v}_{a} \frac{\partial f_{a}^{(0)}}{\partial \epsilon_{a}}, \quad \frac{\partial f_{a}^{(0)}}{\partial \epsilon_{a}}=-\frac{f_{a}^{(0)}}{T}\left(1 \mp f_{a}^{(0)}\right), \\
f_{a}^{(0)}=\frac{1}{e^{\left(\epsilon_{a}-b_{a} \mu_{B}\right) / T} \pm 1},
\end{gathered}
$$

here the single particle energy is denoted as $\epsilon_{a}\left(p_{a}\right)=$ $\sqrt{\vec{p}_{a}^{2}+m_{a}^{2}}, \mu_{B}$ is the baryon chemical potential, $b_{a}$ denotes the baryon number of particle " $a$," e.g., for baryons $b_{\text {baryon }}=1$, for antibaryons $b_{\text {antibaryon }}=-1$ and for mesons $b_{\text {meson }}=0 . \vec{v}_{a}=\vec{p}_{a} / \epsilon_{a}$ is the velocity of the particle. \pm in the equilibrium distribution function is for fermion and boson respectively. The gradient of the equilibrium distribution function $\vec{\nabla} f_{a}^{(0)}$ can be expressed as,

$$
\vec{\nabla} f_{a}^{(0)}=T \frac{\partial f_{a}^{(0)}}{\partial \epsilon_{a}}\left[\epsilon_{a} \vec{\nabla}\left(\frac{1}{T}\right)-b_{a} \vec{\nabla}\left(\frac{\mu_{B}}{T}\right)\right] .
$$

The spatial gradients of temperature and chemical potential can be related using momentum conservation in the system and Gibbs Duhem relation. Momentum conservation leads to $\partial_{i} P=0$. Using Gibbs Duhem relation we then have,

$$
\partial_{i} P=\frac{\omega}{T} \partial_{i} T+T n_{B} \partial_{i}\left(\mu_{B} / T\right)=0 .
$$

Here $\omega=\mathcal{E}+P$ is the enthalpy of the system, $\mathcal{E}$ is the energy density and $P$ is the pressure of the system. The above equation relates the spatial gradient of temperature to the spatial gradient in chemical potential as,

$$
\partial_{i} \mu_{B}=\left(\mu_{B}-\frac{\omega}{n_{B}}\right) \frac{\partial_{i} T}{T} .
$$

Using Eqs. (8) and (6), we have

$$
\vec{\nabla} f_{a}^{(0)}=-\frac{\partial f_{a}^{(0)}}{\partial \epsilon_{a}}\left(\epsilon_{a}-b_{a} \frac{\omega}{n_{B}}\right) \frac{\vec{\nabla} T}{T} .
$$

Using Eq. (9) in Eq. (4) we can write the deviation of the equilibrium distribution function as,

$$
\delta f_{a}=-\tau_{a} \frac{\partial f_{a}^{(0)}}{\partial \epsilon_{a}}\left[q_{a}\left(\vec{E} \cdot \vec{v}_{a}\right)-\left(\epsilon_{a}-b_{a} \frac{\omega}{n_{B}}\right) \vec{v}_{a} \cdot \vec{\nabla} \ln T\right] .
$$

Here we have written, $\vec{\nabla} \ln T=\frac{\vec{\nabla} T}{T}$. Using the deviation from the equilibrium distribution function as given in Eq. (10), the electric current $(\vec{j})$ of the system can be defined as,

$$
\begin{aligned}
\vec{j} & =\sum_{a} g_{a} \int \frac{d^{3} p_{a}}{(2 \pi)^{3}} q_{a} \vec{v}_{a} \delta f_{a} \\
= & \sum_{a} \frac{g_{a}}{3} \int \frac{d^{3} p_{a}}{(2 \pi)^{3}} \tau_{a} q_{a}^{2} v_{a}^{2}\left(-\frac{\partial f_{a}^{(0)}}{\partial \epsilon_{a}}\right) \vec{E} \\
& -\sum_{a} \frac{g_{a}}{3} \int \frac{d^{3} p_{a}}{(2 \pi)^{3}} \tau_{a} q_{a} v_{a}^{2}\left(\epsilon_{a}-b_{a} \frac{\omega}{n_{B}}\right)\left(-\frac{\partial f_{a}^{(0)}}{\partial \epsilon_{a}}\right) \vec{\nabla} \ln T .
\end{aligned}
$$

In Eq. (11), $g_{a}$ is the degeneracy of the particle species " $a$." In the second line of Eq. (11) the integrand depends only on the magnitude of $\vec{p}_{a} \equiv \vec{v}_{a} / \epsilon_{a}$ apart from the factor $v_{a}^{i} v_{a}^{j}$. Therefore one can effectively make the replacement $v_{a}^{i} v_{a}^{j} \rightarrow \frac{1}{3} v_{a}^{2} \delta^{i j}$ for a spherically symmetric system. Further, the sum is over all the baryons and mesons including their antiparticles. For a relativistic system thermal current or the heat current arises corresponding to a conserved particle number. Thermal conduction is defined with reference to the conserved baryon current. Thermal conduction arises when energy flows relative to the baryonic enthalpy. Hence in the presence of conserved baryon current, the heat current of hadron resonance gas can be defined as [4],

$$
\mathcal{I}^{i}=\sum_{a} T_{a}^{0 i}-\frac{\omega}{n_{B}} \sum_{a} b_{a} j_{B_{a}}^{i}
$$

In Eq. (12) $T^{0 i}$ is the "Oi-th" component of the energy momentum tensor $T^{\mu \nu}$. Conserved baryon current is denoted as $j_{B}^{\mu}$ and $n_{B}$ represents the net baryon number density. Using the definition of energy momentum tensor $\left(T^{\mu \nu}\right)$ and baryon current $\left(j_{B}^{\mu}\right)$, heat current $\mathcal{I}^{i}$ as given in Eq. (12) can be recasted as [4],

$$
\begin{aligned}
\mathcal{I}^{i} & =\sum_{a} g_{a} \int \frac{d^{3} p_{a}}{(2 \pi)^{3}} p_{a}^{i} f_{a}-\frac{\omega}{n_{B}} \sum_{a} b_{a} g_{a} \int \frac{d^{3} p_{a}}{(2 \pi)^{3}} v_{a}^{i} f_{a} \\
& =\sum_{a} g_{a} \int \frac{d^{3} p_{a}}{(2 \pi)^{3}} \frac{p_{a}^{i}}{\epsilon_{a}}\left(\epsilon_{a}-b_{a} \frac{\omega}{n_{B}}\right) \delta f_{a} .
\end{aligned}
$$

In Eq. (13) equilibrium distribution function does not contributes because the equilibrium distribution function is a even function of momentum. Using the expression of $\delta f_{a}$ as given in Eq. (10) in Eq. (13) we get for the heat current, 


$$
\begin{aligned}
\mathcal{I}^{i}= & \sum_{a} g_{a} \int \frac{d^{3} p_{a}}{(2 \pi)^{3}} \frac{p_{a}^{i}}{\epsilon_{a}}\left(\epsilon_{a}-b_{a} \frac{\omega}{n_{B}}\right) \delta f_{a} \\
= & \sum_{a} \frac{g_{a}}{3} \int \frac{d^{3} p_{a}}{(2 \pi)^{3}} \tau_{a} q_{a} v_{a}^{2}\left(\epsilon_{a}-b_{a} \frac{\omega}{n_{B}}\right)\left(-\frac{\partial f_{a}^{(0)}}{\partial \epsilon_{a}}\right) \vec{E} \\
& -\sum_{a} \frac{g_{a}}{3} \int \frac{d^{3} p_{a}}{(2 \pi)^{3}} \tau_{a} v_{a}^{2}\left(\epsilon_{a}-b_{a} \frac{\omega}{n_{B}}\right)^{2}\left(-\frac{\partial f_{a}^{(0)}}{\partial \epsilon_{a}}\right) \vec{\nabla} \ln T .
\end{aligned}
$$

The Seebeck coefficient $S$ can be determined using Eq. (11) by setting $\vec{j}=0$, so that the electric field becomes proportional to the temperature gradient and the proportionality factor is nothing but the Seebeck coefficient [83,84]. Hence from Eq. (11) we get,

$$
\vec{E}=S \vec{\nabla} T
$$

here the Seebeck coefficient in the Boltzmann approximation can be identified as,

$$
\begin{aligned}
S & =\frac{\sum_{a} \frac{g_{a}}{3} \int \frac{d^{3} p_{a}}{(2 \pi)^{3}} \tau_{a} q_{a} v_{a}^{2}\left(\epsilon_{a}-b_{a} \frac{\omega}{n_{B}}\right)\left(-\frac{\partial f_{a}^{(0)}}{\partial \epsilon_{a}}\right)}{T \sum_{a} \frac{g_{a}}{3} \int \frac{d^{3} p_{a}}{(2 \pi)^{3}} \tau_{a} q_{a}^{2} v_{a}^{2}\left(-\frac{\partial f_{a}^{(0)}}{\partial \epsilon_{a}}\right)} \\
& =\frac{\sum_{a} \frac{g_{a}}{3 T} \int \frac{d^{3} p_{a}}{(2 \pi)^{3}} \tau_{a} q_{a}\left(\frac{\vec{p}_{a}}{\epsilon_{a}}\right)^{2}\left(\epsilon_{a}-b_{a} \frac{\omega}{n_{B}}\right) f_{a}^{(0)}}{T \sum_{a} \frac{g_{a}}{3 T} \int \frac{d^{3} p_{a}}{(2 \pi)^{3}} \tau_{a} q_{a}^{2}\left(\frac{\overrightarrow{p_{a}}}{\epsilon_{a}}\right)^{2} f_{a}^{(0)}} \equiv \frac{\mathcal{I}_{31} / T^{2}}{\sigma_{e l} / T} .
\end{aligned}
$$

Let us note that at small temperature and large enough baryon chemical potential for which $T s \ll \mu_{B} n_{B}$, $\left(\epsilon_{a}-b_{a} \frac{\omega}{n_{B}}\right) \simeq\left(\epsilon_{a}-b_{a} \mu_{B}\right)$ and in this limit the Seebeck coefficient reduces to the expression for the same given in Ref. [66]. However it may be noted for the system of hadron resonance gas the contribution of pions to entropy can be large and it should not be ignored. We will discuss it more in Sec. IV. The electrical conductivity $\sigma_{e l}$ can be identified from Eq. (11) as,

$$
\sigma_{e l}=\sum_{a} \frac{g_{a}}{3 T} \int \frac{d^{3} p_{a}}{(2 \pi)^{3}} \tau_{a} q_{a}^{2}\left(\frac{\vec{p}_{a}}{\epsilon_{a}}\right)^{2} f_{a}^{(0)},
$$

and the integral $\mathcal{I}_{31}$ in Eq. (16) is,

$\mathcal{I}_{31}=\sum_{a} \frac{g_{a}}{3 T} \int \frac{d^{3} p_{a}}{(2 \pi)^{3}} \tau_{a} q_{a}\left(\frac{\overrightarrow{p_{a}}}{\epsilon_{a}}\right)^{2}\left(\epsilon_{a}-b_{a} \frac{\omega}{n_{B}}\right) f_{a}^{(0)}$.

We would like to make two comments regarding the expression for the Seebeck coefficient as given in Eq. (16). First, the Seebeck coefficient can be positive and negative as the numerator depends linearly on electric charge while the integrand itself is not manifestly positive definite. Second, in the numerator only the baryons will contribute as the mesonic contribution will cancel out. In terms of $\sigma_{e l}$ and $S$ the electric current can be expressed as,

$$
\vec{j}=\sigma_{e l} \vec{E}-\sigma_{e l} S \vec{\nabla} T .
$$

In a similar way, the heat current as given in Eq. (14) can be expressed as,

$$
\overrightarrow{\mathcal{I}}=T \sigma_{e l} S \vec{E}-k_{0} \vec{\nabla} T
$$

where $k_{0}$ is the thermal conductivity and is expressed as [4],

$k_{0}=\sum_{a} \frac{g_{a}}{3 T^{2}} \int \frac{d^{3} p_{a}}{(2 \pi)^{3}} \tau_{a}\left(\frac{\vec{p}_{a}}{\epsilon_{a}}\right)^{2}\left(\epsilon_{a}-b_{a} \frac{\omega}{n_{B}}\right)^{2} f_{a}^{(0)}$.

Using Eqs. (19) and (20), we can express the heat current $\overrightarrow{\mathcal{I}}$ in terms of electric current $\vec{j}$ in the following way,

$$
\overrightarrow{\mathcal{I}}=T S \vec{j}-\left(k_{0}-T \sigma_{e l} S^{2}\right) \vec{\nabla} T .
$$

From Eq. (22) we can identify the Peltier coefficient (П) and thermal conductivity $(k)$ in the presence of nonvanishing Seebeck coefficient as,

$$
\begin{gathered}
\Pi=T S, \\
k=k_{0}-T \sigma_{e l} S^{2} .
\end{gathered}
$$

Note that the Peltier coefficient $(\Pi)$ in terms of the Seebeck coefficient $(S)$ as given in Eq. (23) can be considered as the consistency relation which can also be obtained using the Onsager relation [85]. Also, note that from Eq. (24) the thermal conductivity in the absence of any thermoelectric effect matches the expression of the thermal conductivity as given in Ref. [4]. Here some comments on the formalism as given earlier in Ref. [66] is in order. The basic difference between the expression of the total Seebeck coefficient as given in Eq. (16) for a multicomponent system and the same as given in Ref. [66], is the presence of the factor $\omega / n_{B}$. In Ref. [66] instead of the factor $\omega / n_{B}$, baryon chemical potential $\mu_{B}$ enters into the expression of the Seebeck coefficient. This is because in Ref. [66] the spatial dependence of $\mu_{B}$ was ignored and the heat current is defined with respect to the baryon chemical potential rather that the conserved baryon current. However similar to Ref. [66], here also the mesonic contributions to the total Seebeck coefficient cancel out in the numerator of the Eq. (16) due to the equal and opposite contribution of the particles and antiparticles. Only baryonic contribution becomes relevant in the numerator at finite $\mu_{B}$. Mesons only contribute to the total enthalpy of the system and also in the total electrical conductivity of the system which enters the denominator of the Eq. (16). This simple picture becomes 
more complicated in the presence of a magnetic field which we discuss in the next section.

\section{MAGNETO-SEEBECK COEFFICIENT AND NERNST COEFFICIENT}

To investigate the effect of a magnetic field on the thermoelectric effect we start with the relativistic Boltzmann transport equation (RBTE) to estimate the deviation from equilibrium $\delta f^{a}$ in the presence of a magnetic field. Under the relaxation time approximation (RTA) using Eq. (1), in the local rest frame the RBTE in the presence of an electric field $(\vec{E})$ and a magnetic field $(\vec{B})$ for a single hadron species can be expressed as $[71,102,103]$,

$$
\frac{\partial f_{a}}{\partial t}+\vec{v}_{a} \cdot \frac{\partial f_{a}}{\partial \vec{r}}+q_{a}\left(\vec{E}+\vec{v}_{a} \times \vec{B}\right) \cdot \frac{\partial f_{a}}{\partial \vec{p}_{a}}=-\frac{\delta f_{a}}{\tau_{a}} .
$$

In a static case where both $f_{a}$ and $f_{a}^{(0)}$ does not depend on time explicitly, the relativistic Boltzmann equation get reduced to [71],

$$
\vec{v}_{a} \cdot \frac{\partial f_{a}}{\partial \vec{r}}+q_{a}\left(\vec{E}+\vec{v}_{a} \times \vec{B}\right) \cdot \frac{\partial f_{a}}{\partial \vec{p}_{a}}=-\frac{\delta f_{a}}{\tau_{a}},
$$

In global thermal equilibrium Eq. (26) is trivially satisfied as both the left-hand side (lhs) and right-hand side (rhs) in Eq. (26) vanishes. Hence, we can express the Boltzmann kinetic equation as given in Eq. (26) as an equation for the deviation from equilibrium distribution function, $\delta f_{a}=f_{a}-f_{a}^{(0)}$. Note that equilibrium distribution function $f_{a}^{(0)}$ does not contribute to the Lorentz force as because $\frac{\partial f_{a}^{(0)}}{\partial \vec{p}_{a}} \propto \vec{v}_{a}$, hence $\left(\vec{v}_{a} \times \vec{B}\right) \cdot \frac{\partial f_{a}^{(0)}}{\partial \vec{p}_{a}}=0$. Only $\delta f_{a}$ contributes to the Lorentz force. Therefore the RBTE in the relaxation time approximation get reduced to,

$\vec{v}_{a} \cdot \frac{\partial f_{a}^{(0)}}{\partial \vec{r}}+q_{a} \vec{E} \cdot \frac{\partial f_{a}^{(0)}}{\partial \vec{p}_{a}}+q_{a}\left(\overrightarrow{v_{a}} \times \vec{B}\right) \cdot \frac{\partial\left(\delta f_{a}\right)}{\partial \vec{p}_{a}}=-\frac{\delta f_{a}}{\tau_{a}}$,

Some comments on the relaxation time approximation in the presence of a magnetic field are in order here. In the relaxation time approximation, the system is not far away from equilibrium due to external perturbation and then it relaxes back toward equilibrium with the characteristics time scale given as the relaxation time. Hence external perturbation is not the dominant scale. For the strongly interacting medium, the strong interaction is responsible for equilibration of the system and the magnetic field is a perturbation with respect to the strong interaction. In the heavy-ion collision, a large magnetic field can be produced at the initial stages. Conservative estimates predict the strength of the magnetic field is of the order of a few $m_{\pi}^{2} \sim 0.02 \mathrm{GeV}^{2}$ at RHIC energies and even less in the low energy collisions. To affect the evolution of the strongly interacting medium the magnetic field should survive for at least several Fermi proper time. Earlier it was thought that the magnetic field is strong for a very short time $\sim 0.2 \mathrm{fm}$ at RHIC energies. This time scale is essentially the passing time of the Lorentz contracted nuclei. Subsequently the magnetic field rapidly decays $[13,104]$. However it was pointed out by Tuchin in Ref. [19] that due to induced currents, the magnetic field does not decay very rapidly in a conducting medium, rather the magnetic field satisfies a diffusion equation with the diffusion constant equal to $1 /\left(\sigma_{e l} \tilde{\mu}\right)[18]$. Here $\tilde{\mu}$ is the magnetic permeability, not to be confused with the baryon chemical potential, and $\sigma_{e l}$ is the electrical conductivity $[105,106]$. Assuming $\tilde{\mu}$ to be $\sim \mathcal{O}(1)$ and considering the lattice QCD estimates of the electrical conductivity $\left(\sigma_{e l} \sim 0.04 T\right)$, it has been argued in Ref. [18] that over a length scale of $\sim 10 \mathrm{fm}$ the magnetic field remains reasonably strong for a timescale of the order of $1 \mathrm{fm}$. With a larger value of the electrical conductivity, the magnetic field can sustain for a longer time scale in the medium. On the other hand, the hadronization time scale and the freezeout time scale (typical time scale is of the order of $\gtrsim 10 \mathrm{fm}$ ) can be large with respect to the time scale of $1 \mathrm{fm}$. Also in the hadronic phase, the value of electrical conductivity can be smaller than the partonic phase $[67,68]$. Interestingly with the magnetic field, the electrical conductivity itself decreases[67,71]. Hence it is expected that at the time of chemical freezeout the strength of the magnetic field may be small in comparison to the initial magnetic field. In the low energy collision, the strength of the initial magnetic field can be even smaller. Using the microscopic transport model (UrQMD) magnetic field created in the noncentral heavy-ion collisions has been estimated in the Ref. [14], for SPS to LHC energies. It has been reported in Ref. [14] that at peak SPS energies the peak strength of the magnetic field can be an order of $e B \sim 0.1 m_{\pi}^{2}$. On the other hand for the RHIC energy scale, the strength of the magnetic field can be $e B \sim m_{\pi}^{2}$ and for the LHC energy scale $e B \sim 15 m_{\pi}^{2}$. However, it is important to note that with an increase in the collision energy the initial magnetic field decays even faster with time [14]. Here we should also mention that in Refs. [15,16] electromagnetic fields have been estimated for the RHIC energy scale, taking into account a finite electrical conductivity as well as chiral magnetic conductivity. These estimates also indicate that a conservative value of the initial magnetic field at RHIC energy scale is $e B \sim m_{\pi}^{2}$. These calculations also show that although in vacuum the initial magnetic field decays very rapidly but due to the presence of a finite electrical conductivity and chiral magnetic conductivity, decay of the initial magnetic field with time is relatively slower in medium. Therefore some part of the initial magnetic field 
can survive in the hadronic phase and it is natural to expect that for the hadronic phase evolution modeled by the HRG model the magnetic field will be small.

Further, if one considers the Bjorken flow solution in transverse relativistic magnetohydrodynamics then the proper time evolution of the magnetic field can be expressed as $e B=e B_{0}\left(\tau_{0} / \tau\right)^{a}$ [107]. In the ideal magnetohydrodynamic (MHD) limit $a=1$ and for dissipative case $a>1$ [107]. If we consider a conservative estimate of the thermalization time $\tau_{0}=1 \mathrm{fm}$ with $e B_{0} \sim m_{\pi}^{2}$, then at $10 \mathrm{fm}$ time scale $e B \sim 0.1 m_{\pi}^{2} \sim 0.002 \mathrm{GeV}^{2}$ in the ideal MHD limit. Note that in ideal MHD limit the value of the electrical conductivity is $\sigma_{e l} \rightarrow \infty$. Hence in the ideal MHD limit, the decay of the magnetic field is very slow. On the other hand for a realistic value of the electrical conductivity $\sigma_{e l}$ the decay of the magnetic field can be even faster, e.g., in Ref. [14] the effect of the conducting medium, i.e., effect of the electrical conductivity has not been taken into consideration for the estimation and the evolution of the magnetic field. In that case, the magnetic field decays very rapidly and becomes very small within the timescale of the order of few Fermi. With an increase in the center of massenergy, the decay of the magnetic field is even faster [14]. The assumption we make here is that for finite electrical conductivity the magnetic field in the hadronic phase is small enough and it does not affect the relaxation time. However, we should keep in mind that this is a result from an analysis of Ref. [107] arising from one dimensional relativistic MHD. A precise value for the strength of the magnetic field on the other hand will require $3+1$ dimensional viscous MHD evolution which is still missing. For some important recent developments in the context of novel analytical and numerical solutions of the relativistic magnetohydrodynamics, e.g., self-similar rotating solutions of non-ideal transverse MHD, $3+1$ dimensional self-similar and Gubser flow solution in ideal relativistic MHD see Refs. [108,109] and also the references therein. In these references boost-invariant as well as nonboost-invariant evolution of the magnetic field has been discussed. Another novel approach using Wigner functions to determine the proper time evolution of various thermodynamic quantities for different parametrizations of the magnetic field evolution, in a uniformly expanding hot magnetized plasma has been attempted in Ref. [110].
Therefore we assume that a small fraction of the initial magnetic field can survive in the hadronic phase and the magnetic field is the subdominant scale. Here we have ignored the effect of the magnetic field in the collision integral as well as in thermodynamics. But if the magnetic field is dominant then one has to carefully consider the effect of the magnetic field in the collision integral as well as in thermodynamics of the system. To solve the RBTE as given in Eq. (27) we take an ansatz to express the deviation of the distribution function from the equilibrium in the following way,

$$
\delta f_{a}=\left(\vec{p}_{a} \cdot \vec{\Xi}\right) \frac{\partial f_{a}^{(0)}}{\partial \epsilon_{a}}
$$

with $\vec{\Xi}$ being related to temperature gradient, electric field, the magnetic field and in general can be written as,

$$
\begin{aligned}
\vec{\Xi}= & \alpha \vec{e}+\beta \vec{h}+\gamma(\vec{e} \times \vec{h})+\rho \vec{\nabla} T \\
& +b(\vec{\nabla} T \times \vec{h})+f(\vec{\nabla} T \times \vec{e}) .
\end{aligned}
$$

In Eq. (29), $\vec{h}=\frac{\vec{B}}{|B|}$ and $\vec{e}=\frac{\vec{E}}{|E|}$, is the direction of the magnetic field and electric field respectively. In the absence of a temperature gradient such an ansatz had been taken earlier in Refs. [67-69,71] which we have generalized to include a temperature gradient. Using Eqs. (28) and (9), RBTE as given in Eq. (27) can be expressed as,

$$
\begin{gathered}
\vec{v}_{a} \cdot\left[-\frac{\partial f_{a}^{(0)}}{\partial \epsilon_{a}}\left(\epsilon_{a}-b_{a} \frac{\omega}{n_{B}}\right) \frac{\vec{\nabla} T}{T}\right]+q_{a}\left(\vec{E} \cdot \vec{v}_{a}\right) \frac{\partial f_{a}^{(0)}}{\partial \epsilon_{a}} \\
-q_{a} B \vec{v}_{a} \cdot(\vec{\Xi} \times \vec{h}) \frac{\partial f_{a}^{(0)}}{\partial \epsilon_{a}}=-\frac{\epsilon_{a}}{\tau_{a}}\left(\vec{v}_{a} \cdot \vec{\Xi}\right) \frac{\partial f_{a}^{(0)}}{\partial \epsilon_{a}} .
\end{gathered}
$$

One should note that in the presence of a magnetic field first law of thermodynamics as well as Gibbs-Duhem relation gets modified. However this modification involves non vanishing magnetization of the system. In this present investigation we are not considering spin-magnetic field interaction and magnetization of the system, hence we are considering Eq. (9) even for a nonvanishing magnetic field. Using the representation of $\vec{\Xi}$ as given in Eq. (29), RBTE as given in Eq. (30), can be expressed as,

$$
\begin{gathered}
q_{a}\left(\vec{E} \cdot \vec{v}_{a}\right)-\alpha q_{a} B \vec{v}_{a} \cdot(\vec{e} \times \vec{h})-\gamma q_{a} B(\vec{e} \cdot \vec{h})\left(\vec{v}_{a} \cdot \vec{h}\right)+\gamma q_{a} B\left(\vec{v}_{a} \cdot \vec{e}\right)-\rho q_{a} B \vec{v}_{a} \cdot(\vec{\nabla} T \times \vec{h})-b q_{a} B(\vec{\nabla} T \cdot \vec{h})\left(\vec{v}_{a} \cdot \vec{h}\right) \\
+b q_{a} B\left(\vec{v}_{a} \cdot \vec{\nabla} T\right)-f q_{a} B(\vec{\nabla} T \cdot \vec{h})\left(\vec{v}_{a} \cdot \vec{e}\right)+f q_{a} B(\vec{e} \cdot \vec{h})\left(\vec{v}_{a} \cdot \vec{\nabla} T\right)-\left(\epsilon_{a}-b_{a} \frac{\omega}{n_{B}}\right)\left(\vec{v}_{a} \cdot \vec{\nabla} \ln T\right) \\
=-\frac{\epsilon_{a}}{\tau_{a}}\left[\alpha\left(\vec{v}_{a} \cdot \vec{e}\right)+\beta\left(\vec{v}_{a} \cdot \vec{h}\right)+\gamma \vec{v}_{a} \cdot(\vec{e} \times \vec{h})+\rho\left(\vec{v}_{a} \cdot \vec{\nabla} T\right)+b \vec{v}_{a} \cdot(\vec{\nabla} T \times \vec{h})+f \vec{v}_{a} \cdot(\vec{\nabla} T \times \vec{e})\right] .
\end{gathered}
$$


Comparing the coefficients of different tensor structures on both sides of Eq. (31) we get,

$$
\begin{gathered}
f=0, \\
q_{a} E+\gamma q_{a} B=-\frac{\epsilon_{a}}{\tau_{a}} \alpha, \\
\gamma=\alpha \tau_{a} \omega_{c_{a}}, \\
\beta=\gamma \tau_{a} \omega_{c_{a}}(\vec{e} \cdot \vec{h})+b \tau_{a} \omega_{c_{a}}(\vec{\nabla} T \cdot \vec{h}), \\
b=\omega_{c_{a}} \tau_{a} \rho, \\
\rho=\frac{\tau_{a}}{\epsilon_{a}} \frac{1}{T}\left(\epsilon_{a}-b \frac{\omega}{n_{B}}\right)-b \tau_{a} \omega_{c_{a}} .
\end{gathered}
$$

$$
\begin{aligned}
\delta f_{a}= & \frac{\tau_{a}}{1+\left(\omega_{c_{a}} \tau_{a}\right)^{2}}\left[q_{a}\left\{\left(\vec{v}_{a} \cdot \vec{E}\right)+\left(\omega_{c_{a}} \tau_{a}\right) \vec{v}_{a} \cdot(\vec{E} \times \vec{h})+\left(\omega_{c_{a}} \tau_{a}\right)^{2}(\vec{E} \cdot \vec{h})\left(\vec{v}_{a} \cdot \vec{h}\right)\right\}\right. \\
& \left.-\frac{\left(\epsilon_{a}-b_{a} \frac{\omega}{n_{B}}\right)}{T}\left\{\left(\vec{v}_{a} \cdot \vec{\nabla} T\right)+\left(\omega_{c_{a}} \tau_{a}\right) \vec{v}_{a} \cdot(\nabla T \times h)+\left(\omega_{c_{a}} \tau_{a}\right)^{2}(\vec{\nabla} T \cdot \vec{h})\left(\vec{v}_{a} \cdot \vec{h}\right)\right\}\right](-) \frac{\partial f_{0_{a}}}{\partial \epsilon_{a}} .
\end{aligned}
$$

Using $\delta f^{a}$ as given in Eq. (40) we can express the electrical current $(\vec{j})$ and the heat current $(\overrightarrow{\mathcal{I}})$ as,

$$
\begin{aligned}
j^{l}= & \sum_{a} g_{a} \int \frac{d^{3} p_{a}}{(2 \pi)^{3}} q_{a} v_{a}^{l} \delta f_{a} \\
= & \sum_{a} \frac{g_{a} q_{a}}{3} \int \frac{d^{3} p_{a}}{(2 \pi)^{3}} \frac{v_{a}^{2} \tau_{a}}{1+\left(\omega_{c_{a}} \tau_{a}\right)^{2}}\left[q_{a} \delta^{l j} E^{j}+q_{a}\left(\omega_{c_{a}} \tau_{a}\right) \epsilon^{l j k} h^{k} E^{j}+q_{a}\left(\omega_{c_{a}} \tau_{a}\right)^{2} h^{l} h^{j} E^{j}\right. \\
& \left.-\left(\epsilon_{a}-b_{a} \frac{\omega}{n_{B}}\right)\left\{\delta^{l j} \frac{\partial \ln T}{\partial x^{j}}+\left(\omega_{c_{a}} \tau_{a}\right) \epsilon^{l j k} h^{k} \frac{\partial \ln T}{\partial x^{j}}+\left(\omega_{c_{a}} \tau_{a}\right)^{2} h^{l} h^{j} \frac{\partial \ln T}{\partial x^{j}}\right\}\right](-) \frac{\partial f_{a}^{(0)}}{\partial \epsilon_{a}},
\end{aligned}
$$

and,

$$
\begin{aligned}
\mathcal{I}^{l}= & \sum_{a} g_{a} \int \frac{d^{3} p_{a}}{(2 \pi)^{3}} v_{a}^{l}\left(\epsilon_{a}-b_{a} \frac{\omega}{n_{B}}\right) \delta f_{a} \\
= & \sum_{a} \frac{g_{a}}{3} \int \frac{d^{3} p_{a}}{(2 \pi)^{3}} \frac{v_{a}^{2} \tau_{a}}{1+\left(\omega_{c_{a}} \tau_{a}\right)^{2}}\left(\epsilon_{a}-b_{a} \frac{\omega}{n_{B}}\right)\left[q_{a} \delta^{l j} E^{j}+q_{a}\left(\omega_{c_{a}} \tau_{a}\right) \epsilon^{l j k} h^{k} E^{j}+q_{a}\left(\omega_{c_{a}} \tau_{a}\right)^{2} h^{l} h^{j} E^{j}\right. \\
& \left.-\left(\epsilon_{a}-b_{a} \frac{\omega}{n_{B}}\right)\left\{\delta^{l j} \frac{\partial \ln T}{\partial x^{j}}+\left(\omega_{c_{a}} \tau_{a}\right) \epsilon^{l j k} h^{k} \frac{\partial \ln T}{\partial x^{j}}+\left(\omega_{c_{a}} \tau_{a}\right)^{2} h^{l} h^{j} \frac{\partial \ln T}{\partial x^{j}}\right\}\right](-) \frac{\partial f_{a}^{(0)}}{\partial \epsilon_{a}}
\end{aligned}
$$

Electric current $(\vec{j})$ and the heat current $(\overrightarrow{\mathcal{I}})$ as given in Eqs. (41) and (42) respectively are quite general. However it is difficult to identify the thermoelectric coefficients from Eqs. (41) and (42). To simplify further calculation, without the loss of generality, we can choose the magnetic field along the $z$ direction and take the electric field $(\vec{E})$, and the temperature gradient $(\vec{\nabla} T)$ perpendicular to it, i.e., in the $x-y$ plane. Under these conditions the components of the electric current in the $x-y$ plane are given as,

$$
\begin{aligned}
j_{x}= & \sum_{a} \frac{g_{a} q_{a}}{3} \int \frac{d^{3} p_{a}}{(2 \pi)^{3}} \frac{v_{a}^{2} q_{a} \tau_{a}}{1+\left(\omega_{c_{a}} \tau_{a}\right)^{2}}\left[E_{x}+\left(\omega_{c_{a}} \tau_{a}\right) E_{y}\right](-) \frac{\partial f_{a}^{(0)}}{\partial \epsilon_{a}} \\
& -\sum_{a} \frac{g_{a} q_{a}}{3 T} \int \frac{d^{3} p_{a}}{(2 \pi)^{3}} \frac{v_{a}^{2} \tau_{a}\left(\epsilon_{a}-b_{a} \frac{\omega}{n_{B}}\right)}{1+\left(\omega_{c_{a}} \tau\right)^{2}}\left[\frac{d T}{d x}+\left(\omega_{c_{a}} \tau_{a}\right) \frac{d T}{d y}\right](-) \frac{\partial f_{a}^{(0)}}{\partial \epsilon_{a}},
\end{aligned}
$$


and,

$$
\begin{aligned}
j_{y}= & \sum_{a} \frac{g_{a} q_{a}}{3} \int \frac{d^{3} p_{a}}{(2 \pi)^{3}} \frac{v_{a}^{2} q_{a} \tau_{a}}{1+\left(\omega_{c_{a}} \tau_{a}\right)^{2}}\left[E_{y}-\left(\omega_{c_{a}} \tau_{a}\right) E_{x}\right](-) \frac{\partial f_{a}^{(0)}}{\partial \epsilon_{a}} \\
& -\sum_{a} \frac{g_{a} q_{a}}{3 T} \int \frac{d^{3} p_{a}}{(2 \pi)^{3}} \frac{v_{a}^{2} \tau_{a}\left(\epsilon_{a}-b_{a} \frac{\omega}{n_{B}}\right)}{1+\left(\omega_{c_{a}}\right)^{2}}\left[\frac{d T}{d y}-\left(\omega_{c_{a}} \tau_{a}\right) \frac{d T}{d x}\right](-) \frac{\partial f_{a}^{(0)}}{\partial \epsilon_{a}} .
\end{aligned}
$$

Equation (43) and (44) can be written in a compact form by introducing the following integrals,

$$
\begin{aligned}
& L_{1_{a}}=\frac{g_{a}}{3} \int \frac{d^{3} p_{a}}{(2 \pi)^{3}} \frac{\tau_{a}}{1+\left(\omega_{c_{a}} \tau_{a}\right)^{2}}\left(\frac{\vec{p}_{a}^{2}}{\epsilon_{a}^{2}}\right)(-) \frac{\partial f_{a}^{(0)}}{\partial \epsilon_{a}}, \\
& L_{2_{a}}=\frac{g_{a}}{3} \int \frac{d^{3} p_{a}}{(2 \pi)^{3}} \frac{\tau_{a}\left(\omega_{c_{a}} \tau_{a}\right)}{1+\left(\omega_{c_{a}} \tau_{a}\right)^{2}}\left(\frac{\vec{p}_{a}^{2}}{\epsilon_{a}^{2}}\right)(-) \frac{\partial f_{a}^{(0)}}{\partial \epsilon_{a}}, \\
& L_{3_{a}}=\frac{g_{a}}{3} \int \frac{d^{3} p_{a}}{(2 \pi)^{3}} \frac{\tau_{a} \epsilon_{a}}{1+\left(\omega_{c_{a}} \tau_{a}\right)^{2}}\left(\frac{\vec{p}_{a}^{2}}{\epsilon_{a}^{2}}\right)(-) \frac{\partial f_{a}^{(0)}}{\partial \epsilon_{a}}, \\
& L_{4_{a}}=\frac{g_{a}}{3} \int \frac{d^{3} p_{a}}{(2 \pi)^{3}} \frac{\tau_{a} \epsilon_{a}\left(\omega_{c_{a}} \tau_{a}\right)}{1+\left(\omega_{c_{a}} \tau_{a}\right)^{2}}\left(\frac{\vec{p}_{a}^{2}}{\epsilon_{a}^{2}}\right)(-) \frac{\partial f_{a}^{(0)}}{\partial \epsilon_{a}} .
\end{aligned}
$$

The integrals as given in Eq. (45)-(48) allow us to write Eqs. (43) and (44), respectively, as

$$
j_{x}=\sum_{a} q_{a}^{2} L_{1_{a}} E_{x}+\sum_{a} q_{a}^{2} L_{2_{a}} E_{y}-\sum_{a} q_{a}\left(L_{3_{a}}-b_{a} \frac{\omega}{n_{B}} L_{1_{a}}\right) \frac{d \ln T}{d x}-\sum_{a} q_{a}\left(L_{4_{a}}-b_{a} \frac{\omega}{n_{B}} L_{2_{a}}\right) \frac{d \ln T}{d y},
$$

and,

$$
j_{y}=\sum_{a} q_{a}^{2} L_{1_{a}} E_{y}-\sum_{a} q_{a}^{2} L_{2_{a}} E_{x}-\sum_{a} q_{a}\left(L_{3_{a}}-b_{a} \frac{\omega}{n_{B}} L_{1_{a}}\right) \frac{d \ln T}{d y}+\sum_{a} q_{a}\left(L_{4_{a}}-b_{a} \frac{\omega}{n_{B}} L_{2_{a}}\right) \frac{d \ln T}{d x} .
$$

Seebeck coefficient in the presence of magnetic field or the magneto-Seebeck coefficient $\left(S_{B}\right)$ in this case can be determined by setting $j_{x}=0$ and $j_{y}=0$, so that the electric field becomes proportional to the temperature gradient. For $j_{x}=0$ and $j_{y}=0$ we can solve Eqs. (49) and (50) to get $E_{x}$ and $E_{y}$ in terms of temperature gradients $\frac{d T}{d x}$ and $\frac{d T}{d y}$ in the following way,

$$
\begin{aligned}
E_{x}= & \frac{\sum_{a} q_{a}^{2} L_{1_{a}} \sum_{a} q_{a}\left(L_{3_{a}}-b_{a} \frac{\omega}{n_{B}} L_{1_{a}}\right)+\sum_{a} q_{a}^{2} L_{2_{a}} \sum_{a} q_{a}\left(L_{4_{a}}-b_{a} \frac{\omega}{n_{B}} L_{2_{a}}\right)}{T\left[\left(\sum_{a} q_{a}^{2} L_{1_{a}}\right)^{2}+\left(\sum_{a} q_{a}^{2} L_{2_{a}}\right)^{2}\right]} \frac{d T}{d x} \\
& +\frac{\sum_{a} q_{a}^{2} L_{1_{a}} \sum_{a} q_{a}\left(L_{4_{a}}-b_{a} \frac{\omega}{n_{B}} L_{2_{a}}\right)-\sum_{a} q_{a}^{2} L_{2_{a}} \sum_{a} q_{a}\left(L_{3_{a}}-b_{a} \frac{\omega}{n_{B}} L_{1_{a}}\right)}{T\left[\left(\sum_{a} q_{a}^{2} L_{1_{a}}\right)^{2}+\left(\sum_{a} q_{a}^{2} L_{2_{a}}\right)^{2}\right]} \frac{d T}{d y}
\end{aligned}
$$

and,

$$
\begin{aligned}
E_{y}= & \frac{\sum_{a} q_{a}^{2} L_{2_{a}} \sum_{a} q_{a}\left(L_{3_{a}}-b_{a} \frac{\omega}{n_{B}} L_{1_{a}}\right)-\sum_{a} q_{a}^{2} L_{1_{a}} \sum_{a} q_{a}\left(L_{4_{a}}-b_{a} \frac{\omega}{n_{B}} L_{2_{a}}\right)}{T\left[\left(\sum_{a} q_{a}^{2} L_{1_{a}}\right)^{2}+\left(\sum_{a} q_{a}^{2} L_{2_{a}}\right)^{2}\right]} \frac{d T}{d x} \\
& +\frac{\sum_{a} q_{a}^{2} L_{1_{a}} \sum_{a} q_{a}\left(L_{3_{a}}-b_{a} \frac{\omega}{n_{B}} L_{1_{a}}\right)+\sum_{a} q_{a}^{2} L_{2_{a}} \sum_{a} q_{a}\left(L_{4_{a}}-b_{a} \frac{\omega}{n_{B}} L_{2_{a}}\right)}{T\left[\left(\sum_{a} q_{a}^{2} L_{1_{a}}\right)^{2}+\left(\sum_{a} q_{a}^{2} L_{2_{a}}\right)^{2}\right]} \frac{d T}{d y} .
\end{aligned}
$$

Equation (51) and (52) can be written in a compact form in the following way, 


$$
\left(\begin{array}{c}
E_{x} \\
E_{y}
\end{array}\right)=\left(\begin{array}{cc}
S_{B} & N B \\
-N B & S_{B}
\end{array}\right)\left(\begin{array}{c}
\frac{d T}{d x} \\
\frac{d T}{d y}
\end{array}\right),
$$

here one can identify the magneto-Seebeck coefficient $\left(S_{B}\right)$ as,

$$
\begin{aligned}
S_{B} & =\frac{\sum_{a} q_{a}^{2} L_{1_{a}} \sum_{a} q_{a}\left(L_{3_{a}}-b_{a} \frac{\omega}{n_{B}} L_{1_{a}}\right)+\sum_{a} q_{a}^{2} L_{2_{a}} \sum_{a} q_{a}\left(L_{4_{a}}-b_{a} \frac{\omega}{n_{B}} L_{2_{a}}\right)}{T\left[\left(\sum_{a} q_{a}^{2} L_{1_{a}}\right)^{2}+\left(\sum_{a} q_{a}^{2} L_{2_{a}}\right)^{2}\right]} \\
& =\frac{\left(\sigma_{e l} / T\right)\left(\mathcal{I}_{31}^{B} / T^{2}\right)+\left(\sigma_{H} / T\right)\left(\mathcal{I}_{42}^{B} / T^{2}\right)}{\left(\sigma_{e l} / T\right)^{2}+\left(\sigma_{H} / T\right)^{2}},
\end{aligned}
$$

and the dimensionless Nernst coefficient [Nernst coefficient $(N)$ times the magnitude of the magnetic field $(B)]$ is given as,

$$
\begin{aligned}
N B & =\frac{\sum_{a} q_{a}^{2} L_{1_{a}} \sum_{a} q_{a}\left(L_{4_{a}}-b_{a} \frac{\omega}{n_{B}} L_{2_{a}}\right)-\sum_{a} q_{a}^{2} L_{2_{a}} \sum_{a} q_{a}\left(L_{3_{a}}-b_{a} \frac{\omega}{n_{B}} L_{1_{a}}\right)}{T\left[\left(\sum_{a} q_{a}^{2} L_{1_{a}}\right)^{2}+\left(\sum_{a} q_{a}^{2} L_{2_{a}}\right)^{2}\right]} \\
& =\frac{\left(\sigma_{e l} / T\right)\left(\mathcal{I}_{42}^{B} / T^{2}\right)-\left(\sigma_{H} / T\right)\left(\mathcal{I}_{31}^{B} / T^{2}\right)}{\left(\sigma_{e l} / T\right)^{2}+\left(\sigma_{H} / T\right)^{2}} .
\end{aligned}
$$

Here we have identified the electrical conductivity in the presence of a magnetic field and the Hall conductivity as, $\sigma_{e l}=\sum_{a} q_{a}^{2} L_{1_{a}}$ and $\sigma_{H}=\sum_{a} q_{a}^{2} L_{2_{a}}$ respectively [67]. The integrals $\mathcal{I}_{31}^{B}$ and $\mathcal{I}_{42}^{B}$ in Eqs. (54) and (55) are defined as $\mathcal{I}_{31}^{B}=\sum_{a} q_{a}\left(L_{3_{a}}-b_{a} \frac{\omega}{n_{B}} L_{1_{a}}\right) \quad$ and $\quad \mathcal{I}_{42}^{B} \equiv$ $\sum_{a} q_{a}\left(L_{4_{a}}-b_{a} \frac{\omega}{n_{B}} L_{2_{a}}\right)$. Note that in the absence of a magnetic field integrals $L_{2_{a}}$ and $L_{4_{a}}$ are identically zero. Hence normalized Nernst coefficient $(N B)$ vanishes in the absence of magnetic field and the magneto-Seebeck coefficient $\left(S_{B}\right)$ boils down to the Seebeck coefficient $(S)$ in the absence of magnetic field as given in Eq. (16). However it is important to note that unlike Eq. (16), in Eq. (54) mesonic contributions do not cancel out in the numerator. In the numerator of Eq. (54) mesonic contributions cancel out in the term $\mathcal{I}_{31}^{B}$ due to opposite contributions of particles and antiparticles. But in the term $\mathcal{I}_{42}^{B}$ mesonic contributions do not cancel out because mesons and its antiparticles contribute equally. Similarly the particles and antiparticles contribute in a constructive way in the Nernst coefficient. For a single baryon species the magnetoSeebeck coefficient and the normalized Nernst coefficient can be expressed as,

$$
S_{B_{a}}=\frac{1}{q_{a} T}\left(\frac{L_{1_{a}} L_{3_{a}}+L_{2_{a}} L_{4_{a}}}{L_{1_{a}}^{2}+L_{2_{a}}^{2}}-b_{a} \frac{\omega}{n_{B}}\right),
$$

and,

$$
N_{a} B=\frac{1}{q_{a} T}\left(\frac{L_{1_{a}} L_{4_{a}}-L_{2_{a}} L_{3_{a}}}{L_{1_{a}}^{2}+L_{2_{a}}^{2}}\right)
$$

respectively. Note that the magneto-Seebeck coefficient and the normalized Nernst coefficient for a single baryon species as given in Eqs. (56) and (57) are very similar to the magneto-Seebeck coefficient and the Nernst coefficient for condensed matter systems [111], apart from the fact that in the condensed matter system $\omega / n_{B}$ factor does not appear rather chemical potential appears in the expression of magneto-Seebeck coefficient. One can also define Peltier coefficient and thermal conductivity in the presence of thermoelectric effect for nonvanishing magnetic field. But due to the presence of magnetic field these expressions become more complicated and we have not discussed it further. Using Eqs. (54) and (55) we can estimate magnetoSeebeck coefficient and normalized Nernst coefficient for hadron resonance gas model by evaluating the integrals $L_{1_{a}}, L_{2_{a}}, L_{3_{a}}$, and $L_{4_{a}}$ as given in Eqs. (45)-(48) respectively. Note that to evaluate these integrals in the HRG model we use the Boltzmann approximation. In the Boltzmann approximation we get,

$$
\begin{aligned}
& L_{1_{a}}=\frac{g_{a}}{3 T} \int \frac{d^{3} p_{a}}{(2 \pi)^{3}} \frac{\tau_{a}}{1+\left(\omega_{c_{a}} \tau_{a}\right)^{2}}\left(\frac{\vec{p}_{a}^{2}}{\epsilon_{a}^{2}}\right) f_{a}^{(0)} \\
& L_{2_{a}}=\frac{g_{a}}{3 T} \int \frac{d^{3} p_{a}}{(2 \pi)^{3}} \frac{\tau_{a}\left(\omega_{c_{a}} \tau_{a}\right)}{1+\left(\omega_{c_{a}} \tau_{a}\right)^{2}}\left(\frac{\vec{p}_{a}^{2}}{\epsilon_{a}^{2}}\right) f_{a}^{(0)}, \\
& L_{3_{a}}=\frac{g_{a}}{3 T} \int \frac{d^{3} p_{a}}{(2 \pi)^{3}} \frac{\tau_{a} \epsilon_{a}}{1+\left(\omega_{c_{a}} \tau_{a}\right)^{2}}\left(\frac{\vec{p}_{a}^{2}}{\epsilon_{a}^{2}}\right) f_{a}^{(0)}, \\
& L_{4_{a}}=\frac{g_{a}}{3 T} \int \frac{d^{3} p_{a}}{(2 \pi)^{3}} \frac{\tau_{a} \epsilon_{a}\left(\omega_{c_{a}} \tau_{a}\right)}{1+\left(\omega_{c_{a}} \tau_{a}\right)^{2}}\left(\frac{\vec{p}_{a}^{2}}{\epsilon_{a}^{2}}\right) f_{a}^{(0)} .
\end{aligned}
$$

The only unknown quantity in the Eqs. (58)-(61) is the relaxation time. In general relaxation time depends on the energy and the momentum of the particles. However for simplicity one takes thermal averaged relaxation time by 
integrating energy dependent relaxation time over equilibrium distribution functions. The thermal averaged relaxation time $\left(\tau_{a}\right)$ of the hadron species " $a$ " in terms of the scattering cross section can be expressed in the following manner [62],

$$
\tau_{a}^{-1}=\sum_{b} n_{b}\left\langle\sigma_{a b} v_{a b}\right\rangle
$$

here $n_{b}$ is the number density of particle "b" and $\left\langle\sigma_{a b} v_{a b}\right\rangle$ represents thermal averaged cross section. The sum in Eq. (62) is over all the hadrons and its resonances. In this case the thermal averaged scattering cross section can be expressed as [62],

$$
\begin{aligned}
\left\langle\sigma_{a b} v_{a b}\right\rangle= & \frac{\sigma}{8 T m_{a}^{2} m_{b}^{2} K_{2}\left(m_{a} / T\right) K_{2}\left(m_{b} / T\right)} \\
& \times \int_{\left(m_{a}+m_{b}\right)^{2}}^{\infty} d s \times \frac{\left[s-\left(m_{a}-m_{b}\right)^{2}\right]}{\sqrt{s}} \\
& \times\left[s-\left(m_{a}+m_{b}\right)^{2}\right] K_{1}(\sqrt{s} / T),
\end{aligned}
$$

here $\sigma=4 \pi r_{h}^{2}$ is the total scattering cross section for the hard spheres. $K_{1}$ and $K_{2}$ are the modified Bessel function of first and second order respectively. Using Eqs. (63) and (62) one can calculate the thermal averaged relaxation time. It is important to mention that while the hard sphere scattering cross section $\sigma$ is independent of both temperature and baryon chemical potential, thermal averaged cross section $\langle\sigma v\rangle$ can depend on temperature $(T)$ and chemical potential $\left(\mu_{B}\right)$. This temperature $(T)$ and chemical potential $\left(\mu_{B}\right)$ dependence arise due to the temperature $(T)$ and chemical potential $\left(\mu_{B}\right)$ dependence of the distribution functions. However in Boltzmann approximation $\langle\sigma v\rangle$ is independent of $\mu_{B}$ [112]. After evaluating the thermal averaged relaxation time using Eq. (62) for each hadrons we can estimate the magneto-Seebeck coefficient and Nernst coefficient using Eqs. (54) and (55) respectively.

\section{RESULTS AND DISCUSSIONS}

As we have mentioned earlier, for the hadron resonance gas model with a discrete particle spectrum, we consider here all the hadrons and their resonances up to a mass cutoff $\Lambda=2.6 \mathrm{GeV}$ as is listed in Ref. [113]. A detailed list of particles has been given in Appendix A of Ref. [114]. This apart relaxation time also enters into the calculation of thermoelectric coefficients. For the estimation of relaxation time within the approximation of hard-sphere scattering, we consider a uniform radius of $r_{h}=0.3 \mathrm{fm}$ for all the mesons and baryons $[62,115]$. For these parameters, we estimate the magneto-Seebeck coefficient and the Nernst coefficient using Eqs. (54) and (55) as a function of temperature (T) and baryon chemical potential $\left(\mu_{B}\right)$ for nonvanishing values of the magnetic field.

\section{A. Behavior of Seebeck coefficient with vanishing magnetic field}

In Fig. 1 we show the variation of Seebeck coefficient (S) for vanishing magnetic field with temperature $(T)$ and baryon chemical potential $\left(\mu_{B}\right)$. From Fig. 1 it is clear that for the range of temperature and baryon chemical potential considered in this investigation Seebeck coefficient (S) of the hot and dense hadron resonance gas is negative for vanishing magnetic field. This result is in contrast with our previous work [66]. In Ref. [66] Seebeck coefficient for vanishing magnetic field was found to be positive. The reason behind this discrepancy between the results obtained here and with the results in Ref. [66] is that the formalism adopted in this investigation is different from that of Ref. [66]. For vanishing magnetic field the Seebeck coefficient as given in Eq. (16) is different with respect to the expression of the Seebeck coefficient as obtained in Ref. [66]. In Eq. (16) we have a factor of $\left(\epsilon_{a}-b_{a} \omega / n_{B}\right)$, which is different in Ref. [66] and was $\left(\epsilon_{a}-b_{a} \mu_{B}\right)$. For relativistic systems, heat flow can only be defined relative to a conserved current, for strongly interacting plasma, the heat current is defined with respect to the net baryon current. Let us note that $\omega / n_{B}=T s / n_{B}+\mu_{B}$. For HRG model the entropy arising for pions is large making $T s / n_{B} \gg \mu_{B}$ and in fact overwhelms single particle energy $\epsilon_{a}$. This makes $\left(\epsilon-\omega / n_{B}\right)$ negative for baryons in the HRG model. Baryons have dominant contributions in the Seebeck coefficient for vanishing magnetic field. Hence for the HRG model the factor $\left(\epsilon-\left(\omega / n_{B}\right)\right)$ makes the total

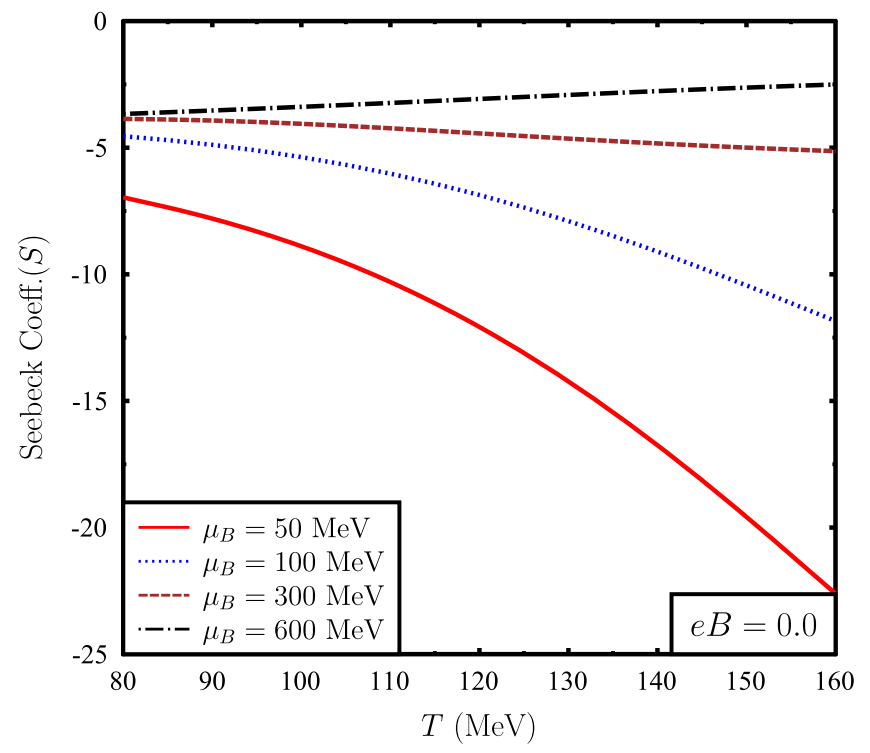

FIG. 1. Variation of Seebeck coefficient $(S)$ for vanishing magnetic field with temperature $(T)$ and baryon chemical potential $\left(\mu_{B}\right)$. With increasing baryon chemical potential $\left(\mu_{B}\right)$, Seebeck coefficient $(S)$ increases. On the other hand with increasing temperature Seebeck coefficient decreases for values of $\mu_{B}(\lesssim 300 \mathrm{MeV})$, but for higher values of baryon chemical potential Seebeck coefficient increases with temperature. 
Seebeck coefficient $(S)$ negative. At this point, it is perhaps relevant to note that the other transport coefficient thermal conductivity $\left(k_{0}\right)$ in the absence of any thermoelectric effect as defined in Eq. (21) also has the term $\left(\epsilon-\left(\omega / n_{B}\right)\right)$. However, in the expression of thermal conductivity $\left(\epsilon-\left(\omega / n_{B}\right)\right)$ always comes as a square, hence in the absence of thermoelectric effect, the positivity of thermal conductivity is ensured which can be seen in Eq. (21). It may be noted that both positive and negative values of the Seebeck coefficient can be found in condensed matter systems, e.g., if for the holes the Seebeck coefficient is positive then for electrons the Seebeck coefficient is negative.

In Fig. 1 it may be observed that with an increase in temperature $(T)$ the Seebeck coefficient $(S)$ decreases for $\mu_{B} \lesssim 300 \mathrm{MeV}$ and it increases with temperature for higher values of $\mu_{B}$. On the other hand with the baryon chemical potential $\left(\mu_{B}\right)$ the Seebeck coefficient $(S)$ always increases. Let us recall that $S=\left(\mathcal{I}_{31} / T^{2}\right) /\left(\sigma_{e l} / T\right)$ therefore variation of the Seebeck coefficient with temperature and baryon chemical potential for vanishing magnetic field can be understood by looking into the behavior of $\mathcal{I}_{31} / T^{2}$ and $\sigma_{e l} / T$ with $T$ and $\mu_{B}$ as shown in Fig. 2. Variation of $\sigma_{e l} / T$ in the absence of magnetic field has been extensively discussed in Ref. [67]. From the left plot in Fig. 2 we can observe that for vanishing magnetic field normalized electrical conductivity $\left(\sigma_{e l} / T\right)$ decrease with temperature and baryon chemical potential. Among all the hadrons mesonic contribution to $\sigma_{e l} / T$ is dominant relative to the baryonic contribution. With increasing temperature and baryon chemical potential mesonic contribution to $\sigma_{e l} / T$ decreases. This decrease of normalized electrical conductivity with temperature and baryon chemical potential is predominantly due to the decrease of relaxation time of mesons with $T$ and $\mu_{B}$ (for a detailed discussion see Ref. [67]). Further from the right plot in Fig. 2 we can see that $\mathcal{I}_{31} / T^{2}$ increases with temperature and baryon chemical potential. This increase of $\mathcal{I}_{31} / T^{2}$ is due to the increasing behavior of $\left(-\omega / n_{B}\right)$ and the equilibrium distribution function with $T$ and $\mu_{B}$ (for a detailed discussion on the variation of $\omega / n_{B}$ see Ref. [68]). For smaller chemical potentials $\mu_{B} \lesssim 300 \mathrm{MeV}$ the decrease of $\sigma_{e l} / T$ is faster compared to the increase of $\mathcal{I}_{31} / T^{2}$ which leads to an increase in magnitude of the ratio that is magnitude of $S$. For higher chemical potentials the increase of $\mathcal{I}_{31} / T^{2}$ due to contributions of baryons becomes faster compared to the decrease of $\sigma_{e l} / T$ leading to the decrease in the magnitude of the Seebeck coefficient. For $\mu_{B} \sim 300 \mathrm{MeV}$ and higher, the increase in $\mathcal{I}_{31} / T^{2}$ with increasing temperature and the decrease in normalized electrical conductivity conspire to give rise to an increase of the Seebeck coefficient with the temperature at high values of baryon chemical potential. Let us recall that in the expression of $\mathcal{I}_{31}$ as given in Eq. (18) mesons do not contribute in the summation. This is because of the opposite electrical charge, the contributions of mesons and its antiparticles cancel exactly in $\mathcal{I}_{31}$. On the other hand in $\sigma_{e l}$ mesonic contribution is nonvanishing. At finite baryon chemical potential baryon contributions are larger compared to the antibaryon contributions due to the equilibrium distribution function. Among all the baryons contribution of proton to $\mathcal{I}_{31} / T^{2}$ is dominant due to its less mass with respect to the other baryons. We can also see from Fig. 1 that with increasing baryon chemical potential
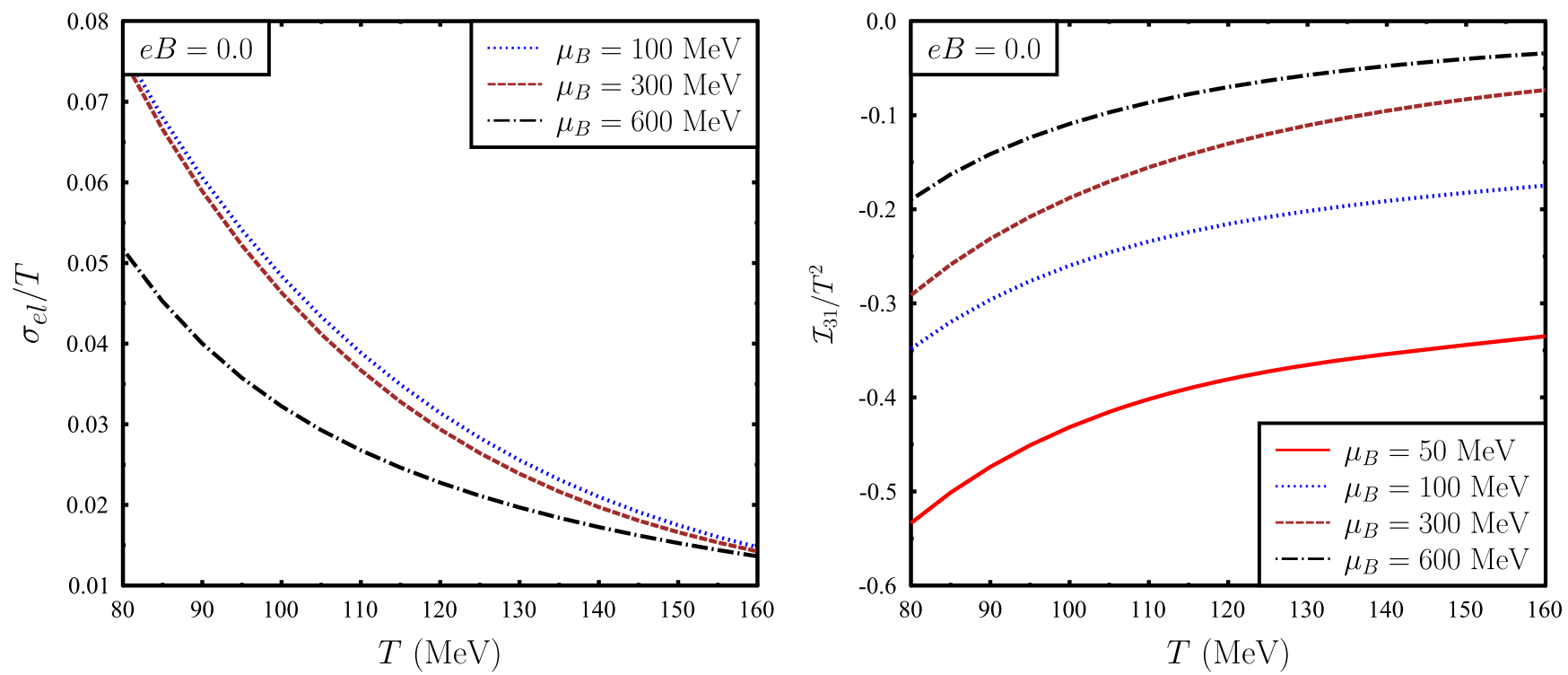

FIG. 2. Left plot: variation of normalised electrical conductivity $\left(\sigma_{e l} / T\right)$ with temperature $(T)$ and baryon chemical potential $\left(\mu_{B}\right)$ for vanishing magnetic field. With increasing $T$ and $\mu_{B}$ normalized electrical conductivity decreases. Right plot: variation of $\mathcal{I}_{31} / T^{2}$ with temperature and baryon chemical potential for vanishing magnetic field. With increasing $T$ and $\mu_{B}, \mathcal{I}_{31} / T^{2}$ increases. 


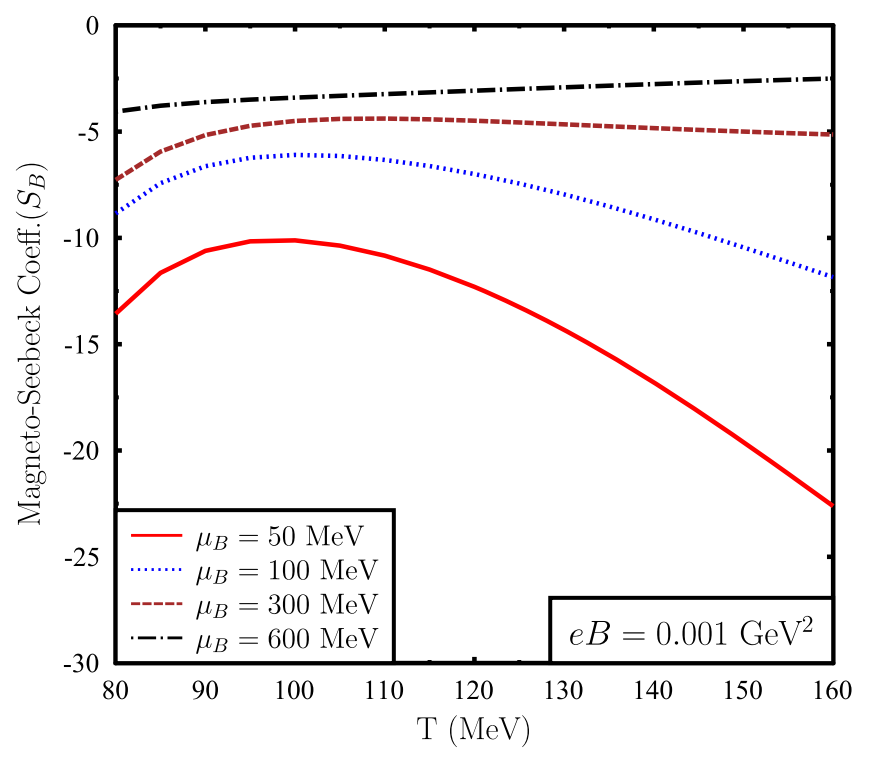

FIG. 3. Variation of the magneto-Seebeck coefficient $\left(S_{B}\right)$ with temperature $(T)$ for different values of baryon chemical potential $\left(\mu_{B}\right)$. With increasing baryon chemical potential $\left(\mu_{B}\right)$ the magneto-Seebeck coefficient $\left(S_{B}\right)$ increases. On the other hand with increasing temperature the magneto-Seebeck coefficient shows nonmonotonic behavior for $\mu_{B} \lesssim 300 \mathrm{MeV}$ and for higher values of $\mu_{B}$ the magneto-Seebeck coefficient increases.

Seebeck coefficient increases. With increasing baryon chemical potential $\sigma_{e l} / T$ decreases. Among various $\mu_{B}$ dependent factors in $\sigma_{e l} / T$, distribution function increases with $\mu_{B}$ and the relaxation time decreases with $\mu_{B}$ due to large number density of the scatters. It turns out that in HRG model pions are the dominant contributors in the $\sigma_{e l} / T$. Although the number density of pion does not depend on the baryon chemical potential, due to pionnucleon scattering pion relaxation time decreases with $\mu_{B}$, due to which $\sigma_{e l} / T$ decreases with $\mu_{B}$. On the other hand, $\mathcal{I}_{31} / T^{2}$ increases with $\mu_{B}$ as can be seen in Fig. 2. For the range of temperature and baryon chemical potential considered here this variation of $\mathcal{I}_{31} / T^{2}$ and $\sigma_{e l} / T$ with $\mu_{B}$ results in an increasing behavior of the Seebeck coefficient $(S)$ with $\mu_{B}$.

\section{B. Results for magneto-Seebeck coefficient and Nernst coefficient}

Now let us discuss the behavior of Seebeck coefficient in the presence of a nonvanishing magnetic field with temperature and baryon chemical potential. In Fig. 3 we show the variation of the magneto-Seebeck coefficient $\left(S_{B}\right)$ with temperature $(T)$ and baryon chemical potential $\left(\mu_{B}\right)$. Magneto-Seebeck coefficient $\left(S_{B}\right)$ as shown in Fig. 3 shows a nonmonotonic behavior with temperature for $\mu_{B} \lesssim 300 \mathrm{MeV}$. On the other hand for higher values of $\mu_{B}$, magneto-Seebeck coefficient $\left(S_{B}\right)$ increases with temperature. We can also observe that with $\mu_{B}$ magnetoSeebeck coefficient always increases. The expression of the magneto-Seebeck coefficient as given in Eq. (54) is more complicated than its zero magnetic field counterpart. From Eq. (54) we can see that in terms $\mathcal{I}_{31}^{B} / T^{2}$ and $\sigma_{H} / T$, mesonic contribution exactly cancels due to the exact and opposite contribution coming from particles and its antiparticles. But in terms $\mathcal{I}_{42}^{B} / T^{2}$ and $\sigma_{e l} / T$ mesonic contributions do not cancel out. In fact in $\mathcal{I}_{42}^{B} / T^{2}$ and $\sigma_{e l} / T$ mesons are the dominant contributors relative to the baryons. The variation of $S_{B}$ with temperature and baryon chemical potential crucially depend upon the variation of $\sigma_{e l} / T, \sigma_{H} / T, \mathcal{I}_{31}^{B} / T^{2}$ and $\mathcal{I}_{42}^{B} / T^{2}$ with $T$ and $\mu_{B}$. We discuss the variation of these four quantities in what follows.

In the presence of magnetic field the variations of normalized electrical conductivity $\left(\sigma_{e l} / T\right)$ and the Hall conductivity $\left(\sigma_{H} / T\right)$ has been extensively discussed in Ref. [67], but for relatively large values of magnetic field compared to this investigation. For completeness in Fig. 4 we show the variation of $\sigma_{e l} / T$ and $\sigma_{H} / T$. For nonvanishing magnetic field normalized electrical conductivity and the Hall conductivity shows nonmonotonic variation with temperature, basically due to the factors of $\omega_{c} \tau$ in the expressions of $L_{1_{a}}$ and $L_{2_{a}}$, as has been discussed in details in Ref. [67]. From the left plot in Fig. 4 we can see that for low temperature range (below $T \sim 90 \mathrm{MeV}$ ), normalized electrical conductivity increases with $\mu_{B}$, but at higher temperature range it decreases with $\mu_{B}$. In the normalized electrical conductivity there are two contributions, one is due to the mesons and other one is due to the baryons. Mesonic contribution to $\sigma_{e l} / T$ decreases with $\mu_{B}$ on the other hand baryonic contribution increases with $\mu_{B}$. In the low temperature range when the relaxation time is large magnetic field affects the mesonic contribution significantly due to the large value of $\omega_{c} \tau$. But with increasing $\mu_{B}$ the baryonic contribution compensates the decrease in the mesonic contribution and eventually for large $\mu_{B}$, normalized electrical conductivity increases with $\mu_{B}$. However, in the high temperature range the decrease in the mesonic contribution cannot be compensated by the baryonic contribution. Thus in the high temperature range normalised electrical conductivity decreases with $\mu_{B}$. This apart normalised Hall conductivity generically increases with baryon chemical potential as has been discussed in details in Ref. [67]. This increase in the Hall conductivity with $\mu_{B}$ is due to the increasing net baryonic contribution with $\mu_{B}$.

Next we discuss the $T, \mu_{B}$ dependence of the other two quantities $\mathcal{I}_{31}^{B} / T^{2}$ and $\mathcal{I}_{42}^{B} / T^{2}$ on which $S_{B}$ depends. In Fig. 5 it is observed that generically, $\mathcal{I}_{31}^{B} / T^{2}$ as well as $\mathcal{I}_{42}^{B} / T^{2}$ are monotonic functions of temperature and baryon chemical potential at nonvanishing magnetic field. It is clear from the expression of $\mathcal{I}_{31}^{B} / T^{2}$ is that only baryons contribute to $\mathcal{I}_{31}^{B} / T^{2}$ and for baryonic contribution $\omega / n_{B}$ is very large with respect to single particle energy. With increasing temperature and baryon chemical potential the 

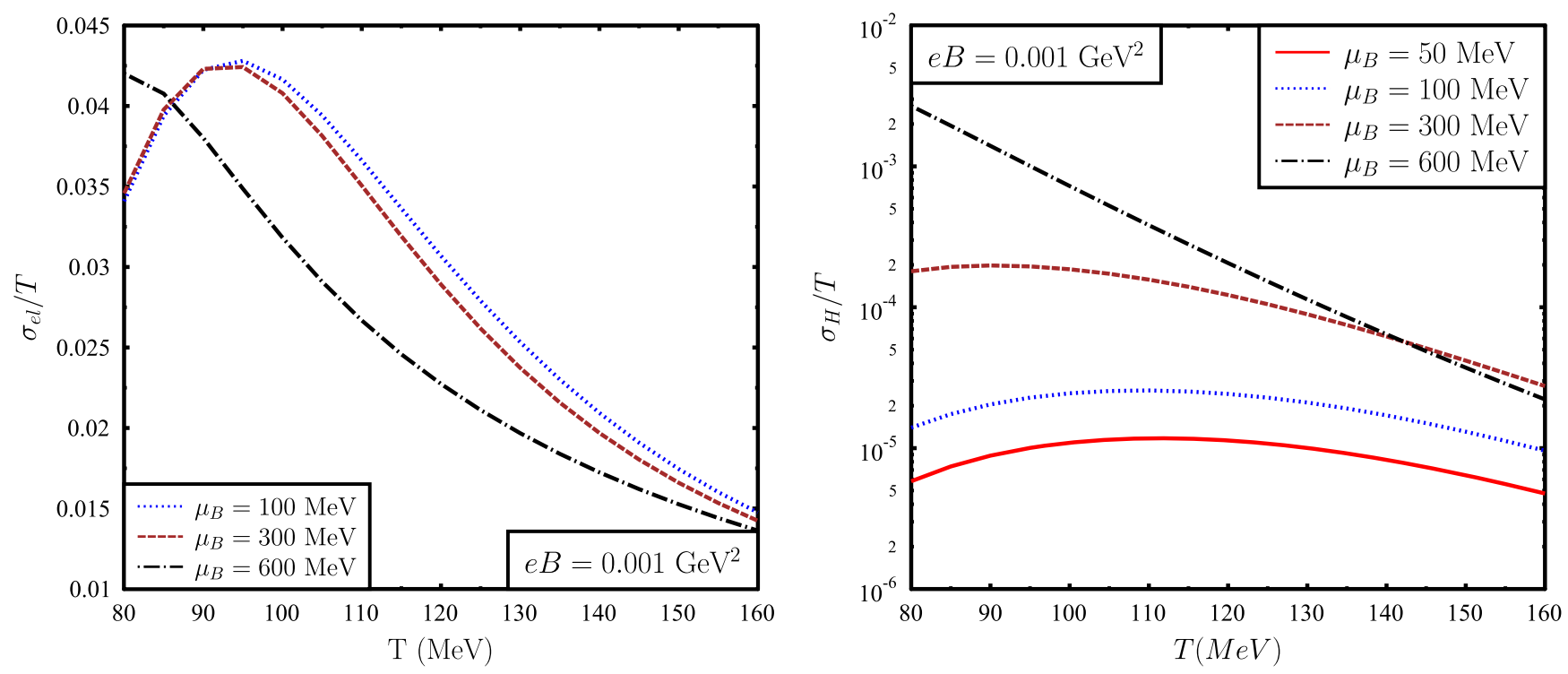

FIG. 4. Left plot: variation of normalized electrical conductivity $\sigma_{e l} / T$ with temperature for different values of baryon chemical potential for nonvanishing magnetic field. For low temperature range $\sigma_{e l} / T$ increases with $\mu_{B}$, otherwise it decreases with baryon chemical potential. With temperature $\sigma_{e l} / T$ shows nonmonotonic variation with a peak structure. Right plot: variation of normalized Hall conductivity $\sigma_{H} / T$ with temperature for different values of baryon chemical potential.

factor $\left(-\omega / n_{B}\right)$ increases along with the increasing distribution function giving rise to increasing behavior of $\mathcal{I}_{31}^{B} / T^{2}$ with temperature and baryon chemical potential. On the other hand in $\mathcal{I}_{42}^{B} / T^{2}$ mesonic contributions becomes dominant with respect to the baryonic contribution. For mesonic contributions, increase in the distribution function with temperature is compensated by the decrease in relaxation time with increasing temperature. Thus with temperature $\mathcal{I}_{42}^{B} / T^{2}$ decreases. Further with increasing baryon chemical potential mesonic contribution decreases because of decrease in the relaxation time. It is important to note that the nonmonotonic nature of the magneto-Seebeck coefficient with temperature has its origin in the nonmonotonic variation of $\sigma_{e l} / T$ and $\sigma_{H} / T$ with temperature.
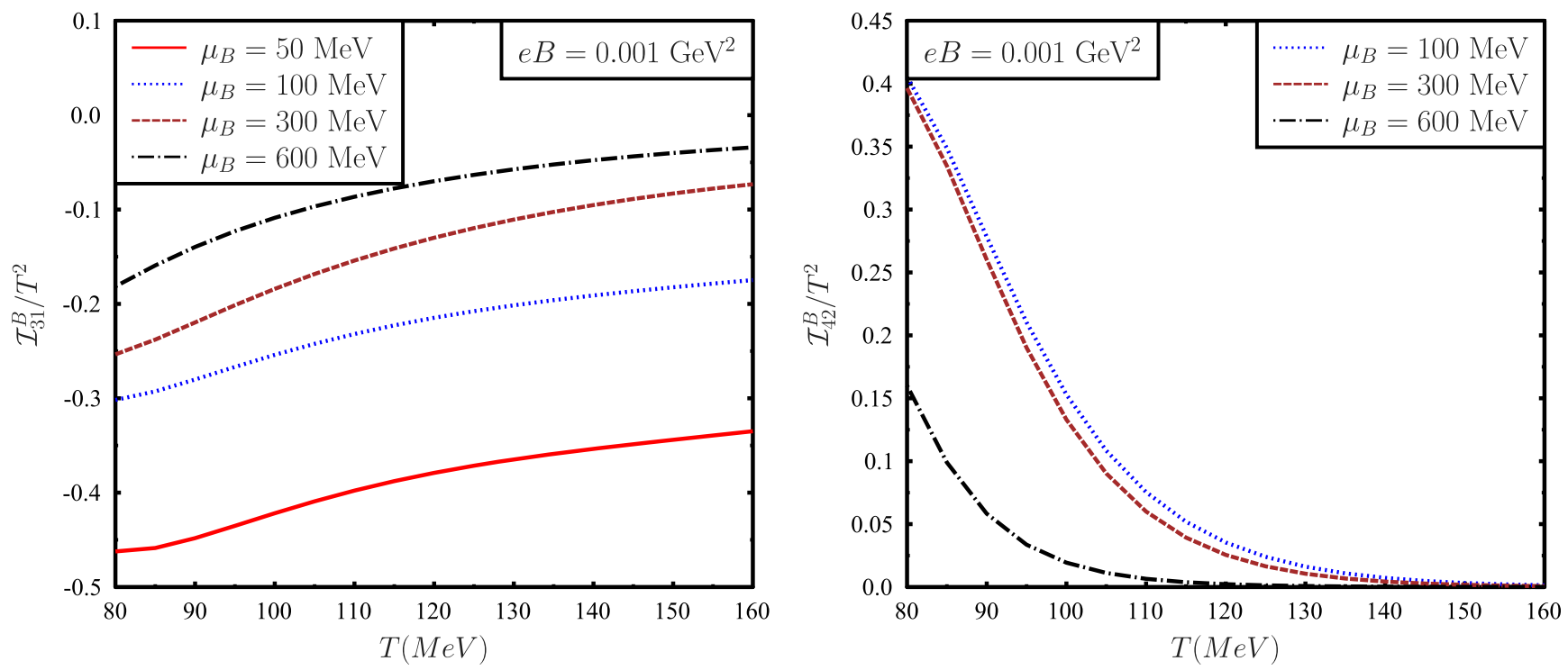

FIG. 5. Left plot: variation of $\mathcal{I}_{31}^{B} / T^{2}$ with temperature for different values of baryon chemical potential for nonvanishing magnetic field. With temperature as well as with baryon chemical potential $\mathcal{I}_{31}^{B} / T^{2}$ increase. Right plot: variation of $\mathcal{I}_{42}^{B} / T^{2}$ with temperature for different values of baryon chemical potential for nonvanishing magnetic field. With increasing temperature as well as with $\mu_{B}, \mathcal{I}_{42}^{B} / T^{2}$ decreases. In $\mathcal{I}_{31}^{B} / T^{2}$ mesonic contributions vanishes due to exact and opposite contributions of the particles and the antiparticles. On the other hand in $\mathcal{I}_{42}^{B} / T^{2}$ mesonic contributions are dominant relative to the baryonic contributions. 



FIG. 6. Left plot: variation of magneto-Seebeck coefficient $\left(S_{B}\right)$ with temperature for different values of magnetic field. With increasing magnetic field $S_{B}$ decreases, however in the high temperature range the effect of magnetic field on $S_{B}$ is less significant. Right plot: variation of magneto-Seebeck coefficient $S_{B}$ with baryon chemical potential for different values of magnetic field. From this plot it is clear that with increasing $\mu_{B}$ magneto-Seebeck coefficient $\left(S_{B}\right)$ increases.

Further we can observe from Fig. 4 that $\sigma_{e l} / T \gg \sigma_{H} / T$ and the order of magnitude values of $\mathcal{I}_{31}^{B} / T^{2}$ and $\mathcal{I}_{42}^{B} / T^{2}$ are of the same order. Therefore the variation of magneto-Seebeck coefficient $S_{B}$ can be approximately expressed as, $S_{B} \sim\left(\mathcal{I}_{31}^{B} / T^{2}\right) /\left(\sigma_{e l} / T\right)$. Thus for small value of baryon chemical potential the maximum in Fig. 3 corresponds to the maximum of $\sigma_{e l} / T$ as shown in Fig. 4 and at high temperature decreasing behavior of $S_{B}$ is due to the decrease of $\sigma_{e l} / T$ with temperature.

Next in Fig. 6 we show the variation of magneto-Seebeck coefficient $\left(S_{B}\right)$ with temperature and baryon chemical potential for different values of the magnetic field. In the relativistic heavy ion collision at the chemical freezeout, in the hadronic phase the center of mass energy $(\sqrt{s})$ dependence of the baryon chemical potential $\left(\mu_{B}\right)$ and temperature can be parametrized as [116],

$$
\mu_{B}(\sqrt{s})=\frac{\tilde{d}}{1+\tilde{e} \sqrt{s}} ; \quad T\left(\mu_{B}\right)=\tilde{a}-\tilde{b} \mu_{B}^{2}-\tilde{c} \mu_{B}^{4} .
$$

here $\sqrt{s}$ in the center of mass-energy, $\tilde{a}=0.166 \pm$ $0.002 \mathrm{GeV} ; \quad \tilde{b}=0.139 \pm 0.016 \mathrm{GeV}^{-1}, \quad \tilde{c}=0.053 \pm$ $0.021 \mathrm{GeV}^{-3}, \quad \tilde{d}=1.308 \pm 0.028 \mathrm{GeV}$ and $\tilde{e}=$ $0.273 \pm 0.008 \mathrm{GeV}^{-1}$. We assume that in the presence of a weak magnetic field the above parametrization of the baryon chemical potential and temperature still holds. It can be shown that for peak RHIC energy scales $(\sqrt{s}=200 \mathrm{~A} \mathrm{GeV})$ the chemical potential at freezeout is $\mu_{B} \sim 30 \mathrm{MeV}$ and the corresponding temperature is $T \sim 178 \mathrm{MeV}$. Further if we consider AGS energy scale $(\sqrt{s}=4.86 \mathrm{~A} \mathrm{GeV})$ then the chemical potential turns out to be $\mu_{B} \sim 580 \mathrm{MeV}$ and the corresponding temperature is $T \sim 120 \mathrm{MeV}$. In our investigation, we have considered the range of temperature and baryon chemical potential of the hadronic phase which covers a wide range of collision energies. Also, we have considered the range of magnetic field which covers a wide range of heavy-ion collisions, from AGS to RHIC energies, e.g., for the RHIC energy scale as we have mentioned above the magnetic field in the hadronic phase can be some fraction of the initial magnetic field. As obtained in the Ref. [14] the peak value magnetic field is of the order of $m_{\pi}^{2}$ or $0.02 \mathrm{GeV}^{2}$. Hence $e B \sim 0.005 \mathrm{GeV}^{2}$ can be a reasonable value of the magnetic field in the hadronic phase.

From Fig. 6 we can observe that with increasing magnetic field the magneto-Seebeck coefficient decreases in the low temperature range $(T \lesssim 120 \mathrm{MeV})$. However in the high temperature range magnetic field does not affect significantly the Seebeck coefficient. Further with baryon chemical potential $S_{B}$ increases for the range of temperature and magnetic field considered in this investigation. The variation of $S_{B}$ with magnetic field can be understood from Eq. (54). In this context we have shown the variation of $\sigma_{e l} / T$ and $\sigma_{H} / T$ with magnetic field in Fig. 7. From the left plot in Fig. 7 we can see that with increasing magnetic field, $\sigma_{e l} / T$ decreases. However in the high temperature range the effect of magnetic field on $\sigma_{e l} / T$ is less significant. The variation of $\sigma_{e l} / T$ with magnetic field has been discussed in great length in Ref. [67]. Basically in the low temperature range magnetic field affect the normalized electrical conductivity because in the low temperature range due to large value of the relaxation time $\omega_{c} \tau$ term in the expression of $\sigma_{e l}$ becomes significant. With increasing magnetic field this 

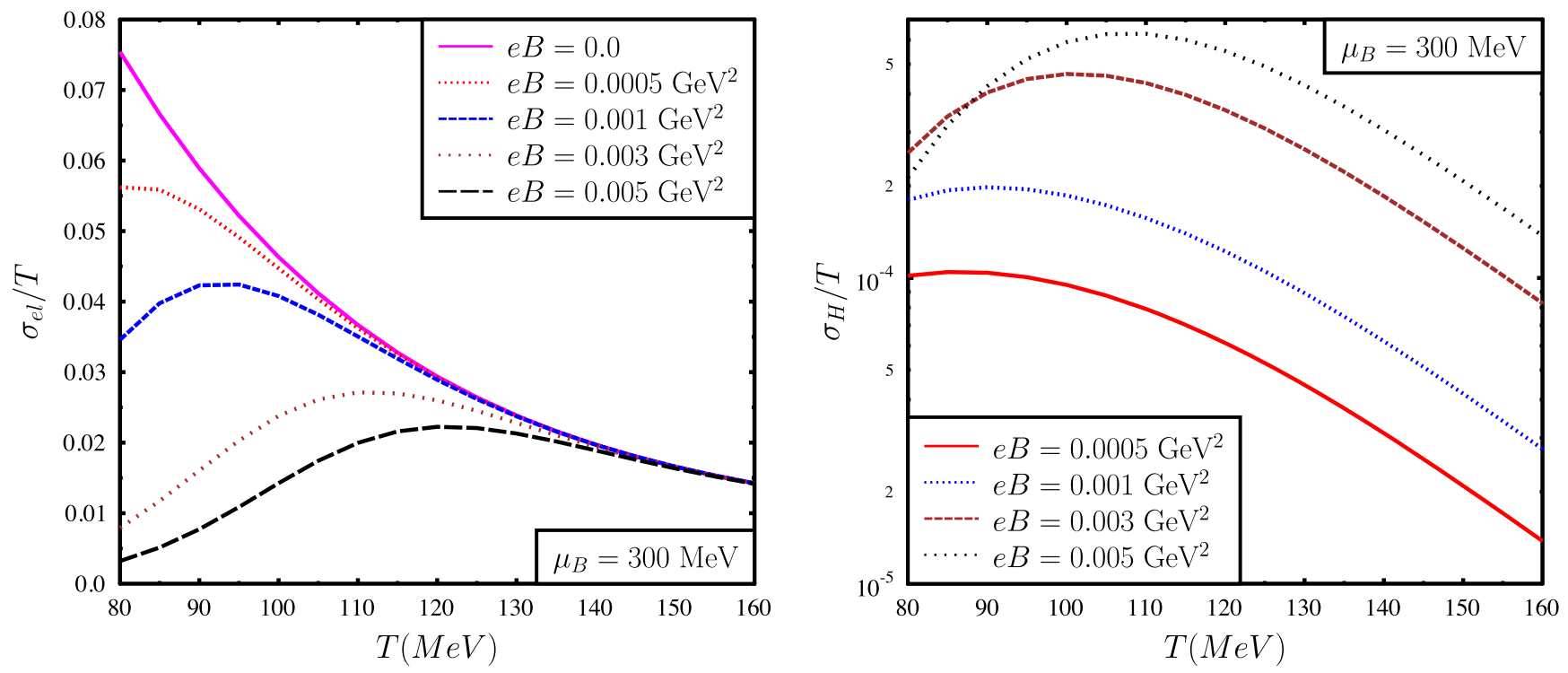

FIG. 7. Left plot: variation of normalized electrical conductivity, $\sigma_{e l} / T \equiv \sum_{a} q_{a}^{2} L_{1_{a}} / T$ with temperature for nonvanishing value of the magnetic field and baryon chemical potential. With magnetic field electrical conductivity generically decrease and with temperature $\sigma_{e l} / T$ shows nonmonotonic behavior. In the high temperature range the effect of magnetic field is less significant on $\sigma_{e l} / T$. Right plot: variation of normalized Hall conductivity $\sigma_{H} / T \equiv \sum_{a} q_{a}^{2} L_{2_{a}} / T$ with temperature for nonvanishing magnetic field. With increasing magnetic field generically Hall conductivity increases for the range of magnetic field considered here.

factor of $\omega_{c} \tau$ is responsible for the decrease in $\sigma_{e l}$. On the other hand in the high temperature range due to very small value of the relaxation time $\omega_{c} \tau$ term remains ineffective. Contrary to $\sigma_{e l} / T$, the normalized Hall conductivity $\left(\sigma_{H} / T\right)$ generically increases with magnetic field. For a detailed discussion on the behavior of $\sigma_{H} / T$ with magnetic field, see Ref. [67].

Further in Fig. 8 we have shown the variation of $\mathcal{I}_{31}^{B} / T^{2}$ and $\mathcal{I}_{42}^{B} / T^{2}$ with magnetic field. With magnetic field $\mathcal{I}_{31}^{B} / T^{2}$ increases. Also in the low temperature range the
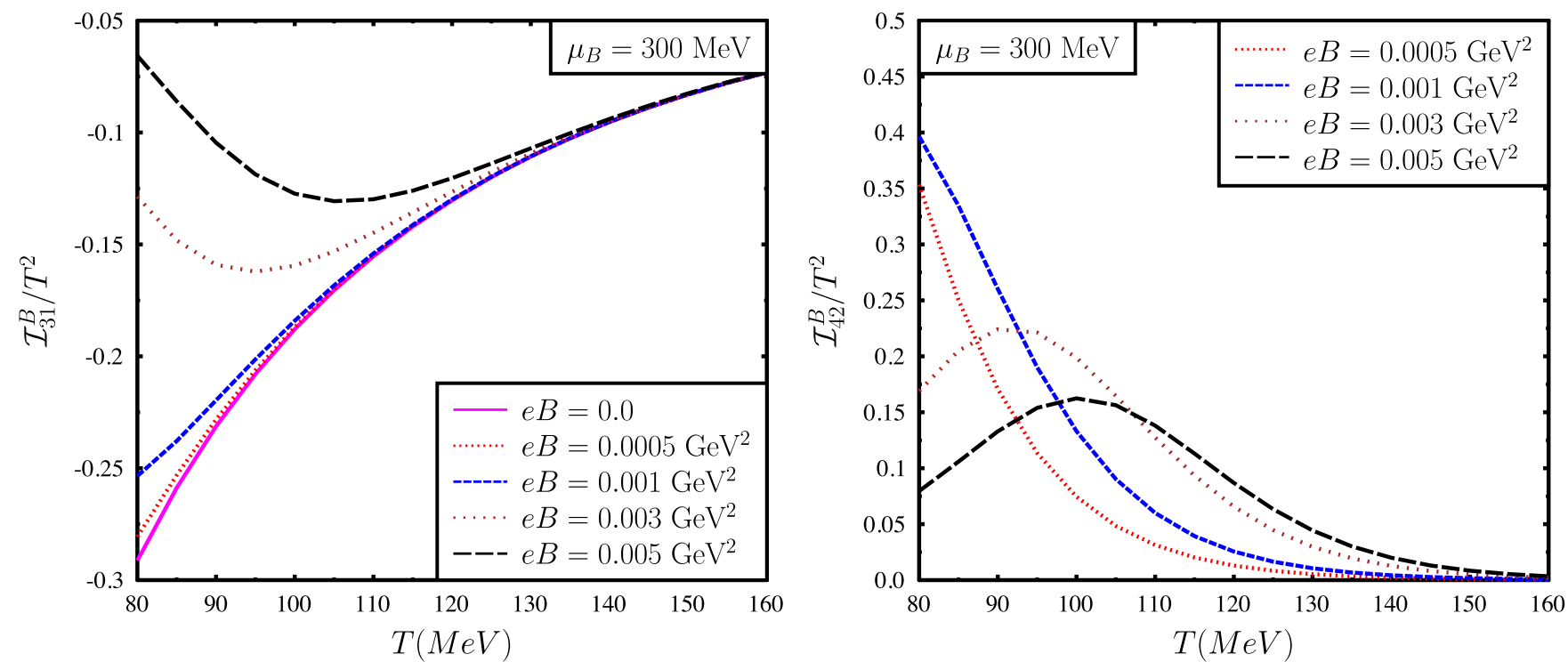

FIG. 8. Left plot: variation of $\mathcal{I}_{31}^{B} / T^{2}$ with temperature at finite baryon chemical potential for nonvanishing values of magnetic field. With increasing magnetic field $\mathcal{I}_{31}^{B} / T^{2}$ increase with respect to its value for vanishing magnetic field. Right plot: variation of $\mathcal{I}_{42}^{B} / T^{2}$ with temperature at finite baryon chemical potential for nonvanishing values of magnetic field. With magnetic field $\mathcal{I}_{42}^{B} / T^{2}$ first increases, then it decreases for higher values of magnetic field. Also for higher values of magnetic field it shows a nonmonotonic variation with temperature for the range of temperature considered here. 



FIG. 9. Left plot: variation of normalised Nernst coefficient $(N B)$ with temperature and magnetic field. With temperature $N B$ decrease and $N B$ increases with increasing magnetic field. However in the high temperature range the effect of magnetic field on $N B$ is less significant. Right plot: Variation of $N B$ with baryon chemical potential $\mu_{B}$ for nonvanishing magnetic field. With baryon chemical potential $N B$ decreases.

effect of magnetic field on $\mathcal{I}_{31}^{B} / T^{2}$ is significant and on the high temperature end due to small value of the relaxation time magnetic field does not affect $\mathcal{I}_{31}^{B} / T^{2}$ significantly. From the right plot in Fig. 8 we observe that with magnetic field first $\mathcal{I}_{42}^{B} / T^{2}$ increases and then with increasing magnetic field it decreases. From Fig. 7 it is clear that $\sigma_{e l} / T$ is order of magnitude larger than $\sigma_{H} / T$. On the other hand the absolute values of $\mathcal{I}_{31}^{B} / T^{2}$ and $\mathcal{I}_{42}^{B} / T^{2}$ does not differ much, which can be observed in Fig. 8. Thus for the situation when $\sigma_{e l} / T$ is larger than $\sigma_{H} / T$ the variation of $S_{B}$ with temperature, chemical potential and magnetic field is predominantly determined by the factors, $\sigma_{e l} / T, \mathcal{I}_{31}^{B} / T^{2}$ and under this condition $S_{B} \sim\left(\mathcal{I}_{31}^{B} / T^{2}\right) /\left(\sigma_{e l} / T\right)$. Hence the variation of $\mathcal{I}_{31}^{B} / T^{2}$ and $\sigma_{e l} / T$ with magnetic field effectively give rise to the variation of $S_{B}$ with magnetic field as has been shown in the left plot in Fig. 6.

Finally we discuss the variation of normalized Nernst coefficient $(N B)$ with temperature and baryon chemical potential for finite magnetic field. In Fig. 9 we show the variation of normalized Nernst coefficient $(N B)$ with temperature and baryon chemical. From Fig. 9 we can observe that with increasing temperature Nernst coefficient $(N B)$ decreases. Due to the order of magnitude difference in the values of $\sigma_{e l} / T$ and $\sigma_{H} / T$ the variation of normalized Nernst coefficient is predominantly determined by $\sigma_{e l} / T$ and $\mathcal{I}_{42}^{B} / T^{2}$. Although $\mathcal{I}_{42}^{B} / T^{2}$ shows a nonmonotonic variation with magnetic field but the normalized Nernst coefficient does not show any nonmonotonic variation with magnetic field, probably because of the fact that with magnetic field $\sigma_{e l} / T$ decrease. This decrease in $\sigma_{e l} / T$ with magnetic field overwhelms the nonmonotonic variation of
$\mathcal{I}_{42}^{B} / T^{2}$. Similarly with baryon chemical potential both $\mathcal{I}_{42}^{B} / T^{2}$ and $\sigma_{e l} / T$ decreases as has been shown in Figs. 4 and (5). But the rapid decrease of $\mathcal{I}_{42}^{B} / T^{2}$ relative to $\sigma_{e l} / T$ possibly give rise to the decreasing behavior of the normalized Nernst coefficient with baryon chemical potential.

\section{CONCLUSIONS}

In heavy-ion collision there is a gradient of temperature from central to peripheral region. This can to lead to a electric field and the relevant Seebeck coefficient as in the condensed matter systems. We had estimated this coefficient within HRG model in Ref. [66]. In that investigation, however, the chemical potential was taken to be uniform. In the present work we have also included the gradient of the baryon chemical potential apart from a spatial gradient in temperature in the estimation of the thermoelectric coefficients. Conservation of momentum of the system along with the Gibbs-Duhem relation are used to relate to the gradient of baryon chemical potential to gradient in temperature. This leads to a modification of the expression for the Seebeck coefficient involving the enthalpy of the system compared to Ref. [66]. Although because of the opposite charges of the mesons, mesonic Seebeck coefficient vanish, nonetheless mesons affect the Seebeck coefficient of the system significantly. This is because the mesonic contribution to the enthalpy of the system is dominant. This leads to a negative Seebeck coefficient of the hadronic system, unlike as in Ref. [66] where the spatial gradient of the chemical potential was not taken into account leading to a positive Seebeck coefficient. 
We have also considered the effects of magnetic field on the thermoelectric properties. Magnetic field leads to a Hall type current due to the Lorentz force. It is observed that the resulting magneto-Seebeck coefficient generically decrease with magnetic field for a given temperature and baryon chemical potential. However at higher temperature and/or chemical potential the magneto-Seebeck coefficient goes over to the Seebeck coefficient at vanishing magnetic field. The induced current perpendicular to the electric field is decided by the Nernst coefficient. It is also observed that the Nernst coefficient for the hadronic matter increases with magnetic field. However at high temperature or baryon chemical potential this approaches the vanishing value for the same at vanishing magnetic field.

In the presence of the thermoelectric coefficient the electrical current $(\vec{j})$ as well as the heat current $(\overrightarrow{\mathcal{I}})$ gets modified. For a nonvanishing value of the Seebeck coefficient the electrical current $\vec{j}=\sigma_{e l} \vec{E}-\sigma_{e l} S \vec{\nabla} T$, has a contribution coming from the Seebeck coefficient $(S)$. Physically the Seebeck coefficient $(S)$ represents the efficiency of any system to convert a temperature gradient to an electrical current. Note that the electrical conductivity $\sigma_{e l}$ is always positive which can be shown using the second law of thermodynamics demanding that in the presence of electromagnetic field $T \partial_{\mu} s^{\mu} \geq 0$, here $s^{\mu}$ is the entropy current [117]. Therefore for a negative Seebeck coefficient, the electric current enhances for a positive temperature gradient. Further if the electric current due to the external electric field and the electric current due to the thermoelectric effect contributes constructively then the net electric current increases. On the other hand the thermal conductivity in the presence of thermoelectric effect, $k=k_{0}-T \sigma_{e l} S^{2}$, indicates that for nonvanishing value of the Seebeck coefficient thermal conductivity decreases. Positivity of the thermal conductivity is required for the theory to be consistent with the second law of thermodynamics, i.e., $T \partial_{\mu} s^{\mu} \geq 0$ [4]. In various literature the positivity of the electrical conductivity and the thermal conductivity has been shown using the formalism of viscous hydrodynamics and viscous magnetohydrodynamics respectively, but the contributions coming from the thermoelectric effects are not considered in these investigations. Therefore it will be interesting to study the effects of thermoelectric coefficients in the entropy production in the context of the viscous hydrodynamics and the magnetohydrodynamics. Phenomenological implication of nonvanishing thermoelectric effect still needs to be explored.

In the present investigation we have focused our attention for hadronic matter. It will definitely be interesting to investigate thermoelectric coefficients for the partonic matter as has been recently attempted in Ref. [118]. In particular it will be interesting and important to investigate the effects of mean field and medium dependent masses on the thermoelectric properties of partonic matter.

\section{ACKNOWLEDGMENTS}

The idea of thermoelectric coefficient in the context of heavy-ion collision arose during a visit of one of the authors (H. M.) to the research group of Prof. Ajit M. Srivastava at Institute of Physics Bhubaneswar. The authors would like to thank Ajit. M. Srivastava for originally suggesting the idea of a thermoelectric coefficient in this context. The authors would like to thank Prof. Jitesh R. Bhatt for useful discussions. The authors would also like to thank Sabyasachi Ghosh, Abhishek Atreya, Aman Abhishek, Chowdhury Aminul Islam, Rajarshi Ray for many discussions on the topic of Seebeck coefficient during working group activities at WHEPP 2017, IISER Bhopal. The work of A. D. is supported by the Polish National Science Center Grant No. 2018/30/E/ST2/00432.
[1] U. W. Heinz and R. Snellings, Annu. Rev. Nucl. Part. Sci. 63, 123 (2013).

[2] P. Romatschke and U. Romatschke, Phys. Rev. Lett. 99, 172301 (2007).

[3] P. K. Kovtun, D. T. Son, and A. O. Starinets, Phys. Rev. Lett. 94, 111601 (2005).

[4] S. Gavin, Nucl. Phys. A435, 826 (1985).

[5] A. Hosoya and K. Kajantie, Nucl. Phys. B250, 666 (1985).

[6] A.Dobado and J. M. Torres-Rincon, Phys. Rev. D 86, 074021 (2012).

[7] C. Sasaki and K. Redlich, Phys. Rev. C 79, 055207 (2009).

[8] C. Sasaki and K. Redlich, Nucl. Phys. A832, 62 (2010).

[9] F. Karsch, D. Kharzeev, and K. Tuchin, Phys. Lett. B 663, 217 (2008).
[10] S. I. Finazzo, R. Rougemont, H. Marrochio, and J. Noronha, J. High Energy Phys. 02 (2015) 051.

[11] A. Wiranata and M. Prakash, Nucl. Phys. A830, 219C (2009).

[12] S. Jeon and L. Yaffe, Phys. Rev. D 53, 5799 (1996).

[13] D. E. Kharzeev, L. D. McLerran, and H. J. Warringa, Nucl. Phys. A803, 227 (2008).

[14] V. Skokov, A. Yu. Illarionov, and V. Toneev, Int. J. Mod. Phys. A 24, 5925 (2009).

[15] H. Li, X. Sheng, and Q. Wang, Phys. Rev. C 94, 044903 (2016).

[16] G. Inghirami, M. Mace, Y. Hirono, L. D. Zanna, D. E. Kharzeev, and M. Bleicher, Eur. Phys. J. C 80, 293 (2020). 
[17] G. Inghirami, L. Del Zanna, A. Beraudo, M. H. Moghaddam, F. Becattini, and M. Bleicher, Eur. Phys. J. C 76, 659 (2016).

[18] A. Das, S. S. Dave, P. S. Saumia, and A. M. Srivastava, Phys. Rev. C 96, 034902 (2017).

[19] K. Tuchin, Phys. Rev. C 83, 017901 (2011); 82, 034904 (2010).

[20] M. Greif, C. Greiner, and G. S. Denicol, Phys. Rev. D 93, 096012 (2016).

[21] M. Greif, I. Bouras, C. Greiner, and Z. Xu, Phys. Rev. D 90, 094014 (2014).

[22] A. Puglisi, S. Plumari, and V. Greco, Phys. Lett. B 751, 326 (2015).

[23] A. Puglisi, S. Plumari, and V. Greco, Phys. Rev. D 90, 114009 (2014).

[24] W. Cassing, O. Linnyk, T. Steinert, and V. Ozvenchuk, Phys. Rev. Lett. 110, 182301 (2013).

[25] T. Steinert and W. Cassing, Phys. Rev. C 89, 035203 (2014).

[26] G. Aarts, C. Allton, A. Amato, P. Giudice, S. Hands, and J.-I. Skullerud, J. High Energy Phys. 02 (2015) 186.

[27] G. Aarts, C. Allton, J. Foley, S. Hands, and S. Kim, Phys. Rev. Lett. 99, 022002 (2007).

[28] A. Amato, G. Aarts, C. Allton, P. Giudice, S. Hands, and J.-I. Skullerud, Phys. Rev. Lett. 111, 172001 (2013).

[29] S. Gupta, Phys. Lett. B 597, 57 (2004).

[30] Y. Burnier and M. Laine, Eur. Phys. J. C 72, 1902 (2012).

[31] H.-T. Ding, A. Francis, O. Kaczmarek, F. Karsch, E. Laermann, and W. Soeldner, Phys. Rev. D 83, 034504 (2011).

[32] O. Kaczmarek and M. Muller, Proc. Sci., LATTICE2013 (2014) 175.

[33] S.-X. Qin, Phys. Lett. B 742, 358 (2015).

[34] R. Marty, E. Bratkovskaya, W. Cassing, J. Aichelin, and H. Berrehrah, Phys. Rev. C 88, 045204 (2013).

[35] D. Fernandez-Fraile and A. Gomez Nicola, Phys. Rev. D 73, 045025 (2006).

[36] Strongly Interacting Matter in Magnetic Field, edited by D. Kharzeev, K. Landsteiner, A. Schmitt, and H. Yee, Lecture Notes in Physics Vol. 871 (Springer-Verlag, Berlin, Heidelberg, 2013).

[37] M. Greif, J. A. Fotakis, G. S. Denicol, and C. Greiner, Phys. Rev. Lett. 120, 242301 (2018).

[38] M. Prakash, M. Prakash, R. Venugopalan, and G. Welke, Phys. Rep. 227, 321 (1993).

[39] A. Wiranata and Madappa Prakash, Phys. Rev. C 85, 054908 (2012).

[40] P. Chakraborty and J. I. Kapusta, Phys. Rev. C 83, 014906 (2011).

[41] A. S. Khvorostukhin, V. D. Toneev, and D. N. Voskresensky, Nucl. Phys. A845, 106 (2010).

[42] S. Plumari, A. Paglisi, F. Scardina, and V. Greco, Phys. Rev. C 86, 054902 (2012).

[43] M. I. Gorenstein, M. Hauer, and O. N. Moroz, Phys. Rev. C 77, 024911 (2008).

[44] J. Noronha-Hostler, J. Noronha, and C. Greiner, Phys. Rev. C 86, 024913 (2012).

[45] S. K. Tiwari, P. K. Srivastava, and C. P. Singh, Phys. Rev. C 85, 014908 (2012).

[46] S. Ghosh, A. Lahiri, S. Majumder, R. Ray, and S. K. Ghosh, Phys. Rev. C 88, 068201 (2013).
[47] R. Lang, N. Kaiser, and W. Weise, Eur. Phys. J. A 51, 127 (2015).

[48] S. Ghosh, G. Krein, and S. Sarkar, Phys. Rev. C 89, 045201 (2014).

[49] A. Wiranata, V. Koch, M. Prakash, and X. N. Wang, J. Phys. Conf. Ser. 509, 012049 (2014).

[50] A. Wiranata, M. Prakash, and P. Chakraborty, Central Eur. J. Phys. 10, 1349 (2012).

[51] A. Tawfik and M. Wahba, Ann. Phys. (Amsterdam) 522, 849 (2010).

[52] J. Noronha-Hostler, J. Noronha, and C. Greiner, Phys. Rev. Lett. 103, 172302 (2009).

[53] G. Kadam and H. Mishra, Nucl. Phys. A934, 133 (2014).

[54] G. Kadam, Mod. Phys. Lett. A 30, 1550031 (2015).

[55] S. Ghosh, Int. J. Mod. Phys. A 29, 1450054 (2014).

[56] N. Demir and A. Wiranata, J. Phys. Conf. Ser. 535, 012018 (2014).

[57] S. Ghosh, Phys. Rev. C 90, 025202 (2014).

[58] J.-B. Rose, J. M. Torres-Rincon, A. Schfer, D. R. Oliinychenko, and H. Petersen, Phys. Rev. C 97, 055204 (2018).

[59] C. Wesp, A. El, F. Reining, Z. Xu, I. Bouras, and C. Greiner, Phys. Rev. C 84, 054911 (2011).

[60] Moritz Greif, Ioannis Bouras, Carsten Greiner, and Zhe Xu, Phys. Rev. D 90, 094014 (2014).

[61] S. A. Bass et al., Prog. Part. Nucl. Phys. 41, 255 (1998).

[62] G. Kadam and H. Mishra, Phys. Rev. C 92, 035203 (2015).

[63] R. K. Mohapatra, H. Mishra, S. Dash, and B. K. Nandi, arXiv:1901.07238.

[64] P. Singha, A. Abhishek, G. Kadam, S. Ghosh, and H. Mishra, J. Phys. G 46, 015201 (2019).

[65] A. Abhishek, H. Mishra, and S. Ghosh, Phys. Rev. D 97, 014005 (2018).

[66] J. R. Bhatt, A. Das, and H. Mishra, Phys. Rev. D 99, 014015 (2019).

[67] A. Das, H. Mishra, and R. K. Mohapatra, Phys. Rev. D 99, 094031 (2019).

[68] A. Das, H. Mishra, and R. K. Mohapatra, Phys. Rev. D 100, 114004 (2019).

[69] A. Das, H. Mishra, and R. K. Mohapatra, Phys. Rev. D 101, 034027 (2020).

[70] J. Dey, S. Satapathy, P. Murmu, and S. Ghosh, arXiv:1907 .11164.

[71] B. Feng, Phys. Rev. D 96, 036009 (2017).

[72] G. S. Denicol, H. Niemi, I. Bouras, E. Molnar, Z. Xu, D. H. Rischke, and C. Greiner, Phys. Rev. D 89, 074005 (2014).

[73] J. I. Kapusta and J. M. Torres-Rincon, Phys. Rev. C 86, 054911 (2012).

[74] H. B. Callen, Thermodynamics (Wiley, New York, 1960).

[75] T. J. Scheidemantel, C. Ambrosch-Draxi, T. Thonhauser, J. V. Badding, and J. O. Sofo, Phys. Rev. B 68, 125210 (2003).

[76] P. Ao, arXiv:cond-mat/9505002; M. Matusiak, K. Rogacki, and T. Wolf, Phys. Rev. B 97, 220501(R) (2018); M. K. Hooda and C.S. Yadav, arXiv:1704.07194; O. Cyr-Choiniere et al., Phys. Rev. X 7, 031042 (2017); L. P. Gaudart, D. Berardan, J. Bobroff, and N. Dragoe, Phys. Status Solidi (RRL) 2, 185 (2008).

[77] S. Sergeenkov, JETP Lett. 67, 680 (1998). 
[78] M. Wysokinski and J. Spalek, J. Appl. Phys. 113, 163905 (2013).

[79] K. P. Wojcik and I. Weymann, Phys. Rev. B 89, 165303 (2014).

[80] K. Seo and S. Tewari, Phys. Rev. B 90, 174503 (2014).

[81] P. Dutta, A. Saha, and A. M. Jayannavar, Phys. Rev. B 96, 115404 (2017); S. Kolenda, M. J. Wolf, and D. Beckmann, Phys. Rev. Lett. 116, 097001 (2016).

[82] M. Shahbazi and C. Bourbonnais, Phys. Rev. B 94, 195153 (2016).

[83] A. Cantarero and F. X. Alvarez, in Thermoelectric Effects: Semiclassical and Quantum Approaches from the Boltzmann Transport Equation, Lecturer Notes in Nanoscale Science and Technology Vol. 16, edited by X. Wang and Z.M. Wang (Springer International Publishing, Switzerland, 2014), ISBN 978-3-319-02011-2.

[84] G. S. Nolas, J. Sharp, and H. J. Goldsmid, in Thermoelectrics: Basic Principles and New Materials Developments, Springer Series in Materials Science Vol. 45 (Springer-Verlag, Berlin Heidelberg, 2001), ISBN 978-3-662-04569-5.

[85] R. Wolfe and G. E. Smith, Phys. Rev. 129, 1086 (1963).

[86] See, "https://www.gsi.de/en/researchaccelerators/fair.htm".

[87] See, "http://nica.jinr.ru".

[88] P. Braun-Munzinger, K. Redlich, and J. Stachel, arXiv: nucl-th/0304013.

[89] A. Andronic, P. Braun-Munzinger, and J. Stachel, Nucl. Phys. A772, 167 (2006).

[90] P. Braun-Munzinger, D. Magestro, K. Redlich, and J. Stachel, Phys. Lett. B 518, 41 (2001); J. Cleymans and K. Redlich, Phys. Rev. C 60, 054908 (1999); F. Becattini, J. Cleymans, A. Keränen, E. Suhonen, and K. Redlich, Phys. Rev. C 64, 024901 (2001); Cleymans, B. Kampfer, M. Kaneta, S. Wheaton, and N. Xu, Phys. Rev. C 71, 054901 (2005); A. Andronic, P. Braun-Munzinger, and J. Stachel, Phys. Lett. B 673, 142 (2009).

[91] R. Dashen, S. Ma, and H. J. Bernstein, Phys. Rev. 187, 345 (1969).

[92] R. Dashen and R. Rajaraman, Phys. Rev. D 10, 694 (1974).

[93] F. Karsch, K. Redlich, and A. Tawfik, Phys. Lett. B 571, 67 (2003).

[94] P. Braun-Munzinger, V. Koch, T. Schafer, and J. Stachel, Phys. Rep. 621, 76 (2016).

[95] M. Nahrgang, M. Bluhm, P. Alba, R. Bellwied, and C. Ratti, Eur. Phys. J. C 75, 573 (2015).

[96] A. Bhattacharyya, S. Das, S. K. Ghosh, R. Ray, and S. Samanta, Phys. Rev. C 90, 034909 (2014).
[97] P. Garg, D. K. Mishra, P. K. Netrakanti, B. Mohanty, A. K. Mohanty, B. K. Singh, and N. Xu, Phys. Lett. B 726, 691 (2013).

[98] A. Bazavov et al., Phys. Rev. D 86, 034509 (2012).

[99] V. V. Begun, M. I. Gorenstein, M. Hauer, V. P. Konchakovski, and O. S. Zozulya, Phys. Rev. C 74, 044903 (2006).

[100] R. K. Mohapatra, Phys. Rev. C 99, 024902 (2019).

[101] D. H. Rischke, M. I. Gorenstein, H. Stocker, and W. Greiner, Z. Phys. C 51, 485 (1991).

[102] S. R. De Groot, W. A. Van Leeuwen, and C. G. Van Weert, Relativistic Kinetic Theory. Principles and Applications (North-Holland, Amsterdam, Netherlands, 1980).

[103] C. Cercignani and G. M. Kremer, The Relativistic Boltzmann Equation: Theory and Applications, Progress in Mathematical Physics Book Series Vol. 22 (Springer, Berlin, 2002), ISBN 978-3-0348-8165-4.

[104] M. Asakawa, A. Majumder, and B. Muller, Phys. Rev. C 81, 064912 (2010).

[105] L. Landau and E. Lifshitz, Electrodynamics of Continuous Media (Pergamon Press, N.Y., USA, 1984), Sec. 58.

[106] J. D. Jackson, Classical Electrodynamics, 3rd ed. (John Wiley \& Sons, Inc., USA, 1999).

[107] V. Roy, S. Pu, L. Rezzolla, and D. Rischke, Phys. Lett. B 750, 45 (2015).

[108] S. M. A. Tabatabaee and N. Sadooghi, Phys. Rev. D 96, 116008 (2017).

[109] M. Shokri and N. Sadooghi, J. High Energy Phys. 11 (2018) 181.

[110] S. M. A. Tabatabaee and N. Sadooghi, Phys. Rev. D 101, 076022 (2020).

[111] Y. Hasegawa, T. Komine, Y. Ishikawa, A. Suzuki, and H. Shirai, Jpn. J. Appl. Phys. 43, 35 (2004).

[112] P. Gondolo and G. Gelmini, Nucl. Phys. B360, 145 (1991).

[113] C. Amsler et al. (Particle Data Group), Phys. Lett. B 667, 1 (2008).

[114] M. Albright, J. Kapusta, and C. Young, Phys. Rev. C 90, 024915 (2014).

[115] P. Braun-Munzinger, I. Heppe, and J. Stachel, Phys. Lett. B 465, 15 (1999).

[116] J. Cleymans, H. Oeschler, K. Redlich, and S. Wheaton, Phys. Rev. C 73, 034905 (2006).

[117] X. G. Huang, M. Huang, D. H. Rischke, and A. Sedrakian, Phys. Rev. D 81, 045015 (2010).

[118] D. Dey and B. K. Patra, arXiv:2004.03149. 